\title{
Robust Linear Beamformer Designs for Coordinated Multi-Point AF Relaying in Downlink Multi-Cell Networks
}

\author{
Chun-I Kuo, Sau-Hsuan Wu, and Chun-Kai Tseng
}

\begin{abstract}
Robust beamforming methods are studied to support relay-assisted coordinated multi-point (CoMP) retransmissions in downlink multi-cell networks. Linear beamformers (BFers) for relay stations of different cells are jointly designed to maintain, in a CoMP amplify-and-forward (AF) relaying manner, the target signal to interference-plus-noise ratios (SINR) at the cellular boundaries of this type of networks. Considering the feasibility in realizations and deployments, BFer designs are only allowed to use the channel state information (CSI) feedbacks of the wireless links inside a network. This kind of designs turns out to be a challenging optimization problem when attempting to maintain the SINR under the estimation and quantization errors in CSI. A conservative criterion and solution method is proposed for this robust design problem. Despite the conservativeness, the proposed method appears to provide an effective BFer design for CoMP AF relaying, either from the perspective of power consumption or from the viewpoints of BFers' complexity and feasibility in syntheses. Simulations also show that when applying the proposed CoMP AF relaying method in Automatic Retransmission reQuest (ARQ), data throughput can be efficiently increased for users close to the joint cellular boundaries inside a multi-cell network.
\end{abstract}

Index Terms-Robust beamforming, CoMP, AF relaying, downlink multi-cell networks, network MIMO.

\section{INTRODUCTION}

$\mathbf{C}$ OORDINATED multipoint (CoMP) transmission with multiple-input multiple-output orthogonal frequency division multiple access (MIMO-OFDMA) is one of the promising concepts to improve spectral efficiency in Long Term Evolution (LTE) or Worldwide Interoperability for Microwave Access (WiMAX) [1], [2]. In addition to CoMP transmission, relaying has also been proposed in these international standards to improve cell coverage and the signal to interferenceplus-noise ratio (SINR) of cell-edge subscriber stations (SSs) [3], [4]. Integrating the concepts of relaying and CoMP transmission, this work studies robust linear beamformer (BFer) designs for relay stations (RSs) to maintain the cell-edge

Manuscript received September 24, 2011; revised February 10, 2012; accepted May 30, 2012. The associate editor coordinating the review of this paper and approving it for publication was C.-C. Chong.

This work was funded by the National Science Council, Taiwan, under Grant NSC 99-2221-E-009-080.

C.-I. Kuo is with MStar Semiconductor, Inc., Taiwan (e-mail: steve.kuo@mstarsemi.com).

S.-H. Wu and C.-K. Tseng are with the Institute of Communications Engineering, Department of Electrical and Computer Engineering, National Chiao Tung University, Hsinchu, Taiwan 300, ROC (e-mail: sauhsuan@cm.nctu.edu.tw, cktseng.cm97g@nctu.edu.tw).

Digital Object Identifier 10.1109/TWC.2012.072512.111764
SINR in a CoMP amplify-and-forward (AF) relaying manner in downlink multi-cell networks. Through the BFer designs, we also examine the effectiveness of CoMP AF relaying in Automatic Retransmission reQuest (ARQ) in such multi-cell wireless networks.

CoMP transmission can be, in general, categorized as inter-site and intra-site CoMPs [1]. Inter-site CoMP performs coordinated transmissions among base stations (BSs) of different cells, while intra-site CoMP only involves coordinations among the different sectors of one cell. Compared to intra-site CoMP, inter-site CoMP is in general much harder to realize due to extra challenges on such as network synchronization, user clustering, backhaul loading, and channel state information (CSI) estimation and feedback accuracy [1]. Nevertheless, inter-site CoMP still attracts significant research attentions as it provides promising gains in system throughput [1]. To fully exploit the potential of inter-site CoMP, BSs should jointly transmit data to multiple SSs. The CoMP system, in this case, becomes a virtual MIMO system wherein both data and CSIs are required to exchange in realtime among BSs [1]. This, however, poses great challenges on the speed and loading of the network that connects the BSs. In view of these constraints, [5] studies coordinated beamforming (BF) that does not require such realtime data exchanges. The BFers discussed throughout this paper basically follow this design methodology in principle.

Despite the throughput potential of inter-site CoMP, the system overhead to estimate and provide CSI at the transmitter (CSIT), and the accompanying estimation and feedback errors make the number of antennas that might be coordinated essentially limited [6]. Besides, to avoid using BF methods that involve complex multi-BS joint processing, limited MIMO network coordination is preferred [7]. Considering these constraints, it seems more practical to form several adjacent cells into a cluster, and a cluster control unit (CU) exists to collect CSIs, synthesize BFers and coordinates coherent transmissions in the cluster. The BFers studied in the sequel are basically designed for this type of CoMP architecture, though not particularly depending on a certain clustering method. Several existing clustering methods can been found in [1], [8]. In particular, field trials in [1] show that significant gain can still be obtained for downlink CoMP in small clusters of largescale cellular networks, in spite of the various aforementioned constraints and impairments in practice. Results in [9] also show that the gain of using CoMP is still larger than the 


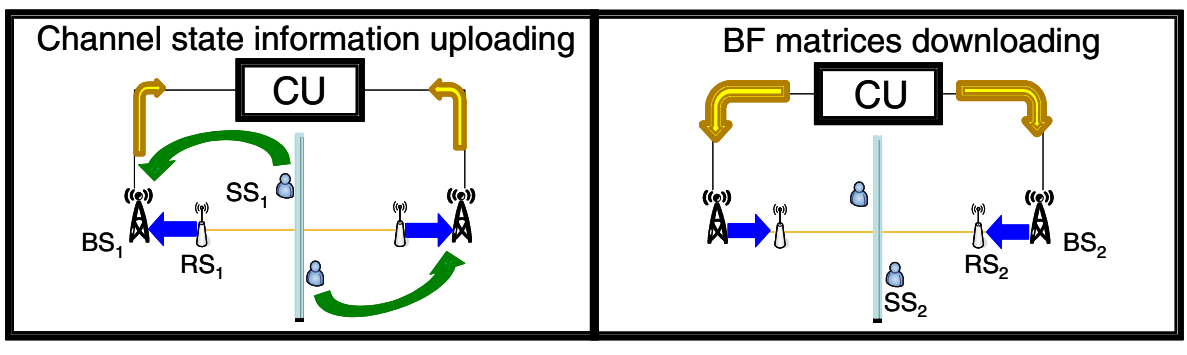

Fig. 1. Illustrations of CSI uploading and BF matrices downloading. The CSIs of BSs, RSs and SSs are exchanged at the CU through their associated BSs.

performance loss from imperfect synchronization even in large multi-cell networks, (according to metrics proposed therein to evaluate the impact of asynchronousness).

Another limiting factor for CoMP transmissions is that the SINR at cellular boundaries is typically low due to the intrinsically weak received signal coming from the associated BS. This makes it difficult to maintain the quality of service (QoS) at cellular boundaries. To overcome this difficulty in single-cell systems, RSs can be used in ARQ to improve the throughput of SSs [4]. In contrast to two-hop relaying [3], the RSs, in this case, retransmit only if the SSs fail to decode data or feed back acknowledgments. Based on this relaying mechanism and the coordinated $\mathrm{BF}$ concept in [5], we study herein BFers designs for inter-site downlink CoMP AF relaying used in ARQs. The presented methods can be extended for two-hop relaying as well. More complete discussions about the architecture, feasibility and effectiveness of relay technologies in LTE-Advanced can be found in [2], [4], [10], [11].

Considering that resources allocated for information exchanges are likely to be limited between RSs of different cells, we choose to study CoMP AF BF schemes that require CSI feedbacks from RSs and SSs only. Since no realtime data exchange is needed among BSs or RSs, this design criterion also reduces the algorithm's dependence on precise network synchronization. Nevertheless, to suppress multiple access interference (MAI), both BSs and RSs need to have multiple antennas no less than the number of cells, and the $\mathrm{CU}$ that synthesizes the BFers needs to have the full downlink CSIs between the BSs, RSs and SSs for the cluster of interest.

The CSI available at the CU are subject to uncertainties from channel estimation, feedback delay and quantization errors. With small amount of bits reserved for CSI feedbacks and advanced estimation schemes to be used in the emerging nextgeneration cellular networks, quantization errors and feedback delays are likely to be the dominant sources of uncertainties in CSIs [12]. To compensate the performance degradation due to CSI feedbacks errors, we adopt a bounded uncertainty model for CSI at the CU [13]. According to this uncertainty model, we design robust linear BFers for RSs to minimize the total transmit power that satisfies the SINR requirement of each single SS for all uncertainties within a specified region. The uncertainties considered in this kind of worstcase designs do not necessarily require statistical models, and, hence, may incorporate other system uncertainty factors, e.g. estimation errors, in CSI as well. Robust designs based on similar uncertainty models for typical CoMP systems or relaying can also be found in [12], [14]-[17].

In contrast to the rich results in robust designs for typical AF relaying [16], [17] or downlink CoMP [12], few literatures discuss CoMP relaying [18] or CoMP AF relaying [19]. To the best of our knowledge, robust BFers for CoMP AF relaying have not been studied before in the proposed context. The BFer designs appear to be a challenging optimization problem. The difficulties mainly lie in the coupled BS-to-RS and RS-to-SS CSI uncertainties in AF relaying. To alleviate the difficulties, we seek for low-complexity suboptimal designs to construct the robust BFers and evaluate the impact of the uncertainties on BFers' performance. To this end, we first formulate a semi-robust design criterion that ignores the BSto-RS CSI uncertainties. Based on this criterion, we then establish the necessary and rank-one condition for the optimal BF matrices, making use of the S-procedure [20]. This rankone structure greatly reduces the complexities in BFer designs. Using a semi-definite relaxation (SDR) procedure similar to those in [21], the robust design problem can further be transformed into a semidefinite programming (SDP) problem, and then be solved with the commercialized optimization solvers such as CVX or SeDuMi [22], [23].

Though elegant, the above SDR approach does not provide an explicit structure for BFers, and cannot be extended to handle the full robust design problems considered herein. Alternatively, we develop another approach for the semi-robust design problem based on a two-tier iterative procedure of quadratic constrained quadratic programming (QCQP). The resultant BFers possess near closed-form expressions and have a lower complexity in realization. Simulation results also show that the BFers present a performance very close to the SDR one, in the senses of power consumption and BFer's feasibility. More importantly, the proposed QCQP scheme can be extended to solve a conservative but full robust design that accounts for the BS-to-RS CSI uncertainties as well. According to our simulations, the performance degradations due to the extra uncertainties in the BS-to-RS CSI is very limited. And the downlink throughput close to the joint cellular boundaries can be effectively improved, when applying the proposed BFers and CoMP AF relaying methods to ARQ in the simulated multi-cell networks. Although the results are examined from a physical layer point of view, and the optimal solution for the full robust design is still an open problem, considering the tradeoff between the performance and feasibility in BFer syntheses, the proposed CoMP AF relaying method seems to provide an effective compromise in maintaining the SINR, and hence the QoS, in CoMP systems. 


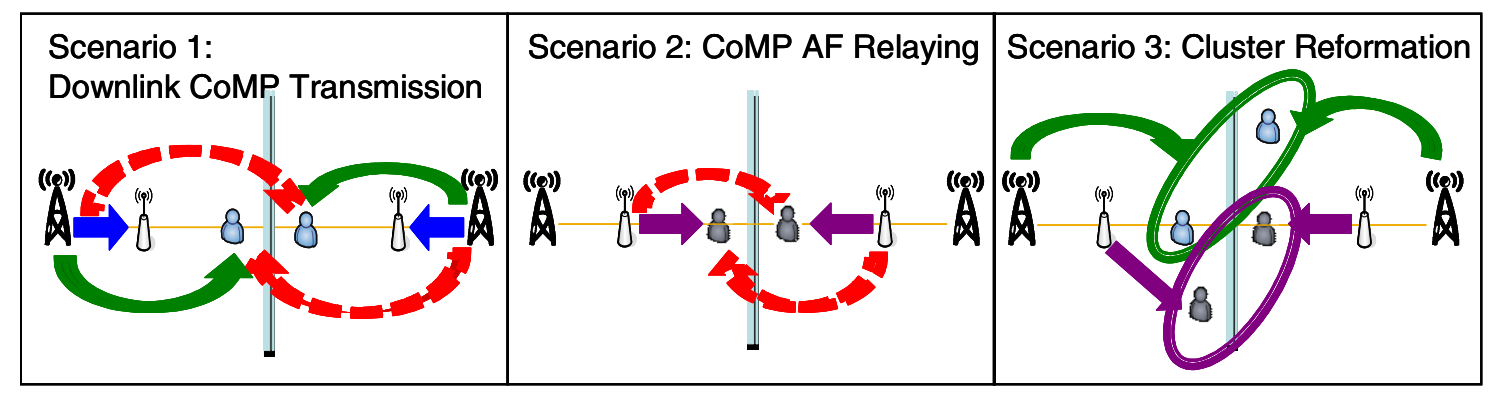

Fig. 2. Operating scenarios of relay-assisted downlink CoMP transmissions. In scenario 1, BSs perform CoMP transmissions to SSs in a cluster. Green arrows represent signals for the intended SSs, blue arrows stand for signals overheard by RSs and red arrows are cross-cell MAI. In scenario 2, both of the SSs fail to decode data and issue CoMP AF relaying for ARQ. In scenario 3, SSs are reformed into two different clusters, one for ARQ and the other for downlink CoMP transmissions.

Notations: We follow the conventions to use, e.g. ' $a$ ' to represent a scalar, ' $a$ ' to represent a vector and ' $\mathbf{A}$ ' to represent a matrix. Besides, $\|\cdot\|$ represents the vector Euclidean norm. The superscripts ' $T$ ', ' $H$ ' and ' -1 ' represent the transpose, Hermitian transpose and inverse of matrices, respectively. And $\mathbf{A} \succeq 0$ means that matrix $\mathbf{A}$ is positive semidefinite. The notations $\mathbb{C}^{i \times j}$ and $\mathbb{R}^{i \times j}$ represent the sets of $i \times j$-dimensional complex and real matrices. For simplicity, $\forall i \triangleq\{i=1 \ldots N\}$ and $\forall j \neq i \triangleq\{i, j=1 \ldots N, j \neq i\}$.

\section{System Model and Problem Statement for COMP AF RELAYING}

We consider a CoMP system that exists a CU to collect CSIs feedbacks, synthesize BFers and coordinate transmissions and retransmissions in a cluster [1]. Training signals are sent by the participating BSs and RSs regularly, from which RSs and SSs can estimate the downlink BS-to-RS, BS-to-SS and RS-to-SS CSIs, respectively. The CSI estimates are quantized and fed back through their associated BSs to the CU. The BSs and RSs are later informed of their own BF vectors or matrices via their associated BSs. A diagram illustrating such a coordination procedure for a two-cell cluster is presented in Fig. 1.

When employing downlink CoMP transmission and $\mathrm{AF}$ relaying, for instance, in a two-cell network in Figs. 1 and 2 , the CU first chooses two SSs from different cells to form a cluster and then coordinates the BSs of the two cells to do downlink CoMP data transmissions to the two SSs. At the end of a transmission round, the SSs decode the packets, and detect and feed back whether there are errors in their packets. If both of the SSs find errors, the CU will coordinate the RSs, through their BSs, to resend the data in a CoMP AF relaying manner. In case only one SS of the cluster detects errors, the system may need to reform a new cluster for CoMP relay retransmissions, and a new cluster for the other SS as well if the SS does not have packets for retransmissions in its associated RS. In case of no error, the CoMP transmission continues if needed.

Considering the limitation on the number of antennas that can be jointly coordinated (either across BSs or RSs), the $\mathrm{BFer}$ design for downlink CoMP AF relaying is studied for a small cluster that consists of $N$ cells, with each of which consists of a single BS and a RS. To enhance the received signal quality near cellular boundaries, RSs are considered only located between the BSs and SSs [24] as shown in Figs. 1 and 2, and are, thus, in a fair distance from their joint boundaries. Under this geometrical relationship, interferences to RSs from signals of the BSs of adjacent cells are likely to be very small and are, thus, ignored in the subsequent BFer designs. The complexity of BFer designs can be much reduced due to this simplification. A similar approach has also been adopted in [25] to seek for a proper balance between BFers' performance and CSI feedback overheads in typical downlink CoMP systems. The effects due to ignoring this type of crosscell BS-to-RS MAI will be assessed later by simulations to verify the proposed BFer design methods.

\section{A. System and Signal Models}

Under the system architecture introduced previously, RSs only account for signals from their associated BSs. Neverthelss, a $\mathrm{SS}_{i}$ close to the edge of its cell $\mathrm{C}_{i}$, will receive its desired signal from $\mathrm{BS}_{i}$ or its associated $\mathrm{RS}_{i}$, and meanwhile picks up interferences from $\mathrm{BS}_{j}$ or $\mathrm{RS}_{j}, \forall j \neq i$. To suppress the interferences in CoMP transmissions without data sharing among the BSs or RSs, each BS or RS should be equipped with at least $N$ antennas, while each SS may have only one antenna. For simplicity, we assume in this paper that each BS or RS has exactly $N$ antennas, and each SS has one antenna only. The results obtained herein can be easily extended to cases with more antennas.

According to the above system setting, the signal received at $\mathrm{RS}_{i}$ can be modeled as

$$
\mathbf{y}_{r_{i}}=\mathbf{h}_{b_{i}, r_{i}} x_{i}+\mathbf{n}_{r_{i}}
$$

where $x_{i}$ stand for the data symbols of unit power sent for $\mathrm{SS}_{i}$ from $\mathrm{BS}_{i}$, and $\mathbf{h}_{b_{i}, r_{i}} \triangleq \mathbf{H}_{b_{i}, r_{i}} \mathbf{w}_{b_{i}}$ in which $\mathbf{H}_{b_{i}, r_{i}} \in \mathbb{C}^{N \times N}$ are the channel matrices from $\mathrm{BS}_{i}$ to $\mathrm{RS}_{i}$ and $\mathbf{w}_{b_{i}} \in \mathbb{C}^{N \times 1}$ are the BF vectors for $\mathrm{BS}_{i}$. The noise vectors $\mathbf{n}_{r_{i}} \in \mathbb{C}^{N \times 1}$ are Gaussian distributed with zero-mean and variance equal to $\sigma^{2} \mathbf{I}_{N}$, denoted by $\sim \mathcal{C N}\left(\mathbf{0}, \sigma^{2} \mathbf{I}_{N}\right)$, and the entries of $\mathbf{H}_{b_{i}, r_{i}}$ are modeled as flat Rayleigh faded. Given $\mathbf{w}_{b_{i}}, \mathbf{h}_{b_{i}, r_{i}} \in \mathbb{C}^{N \times 1}$ can be regarded as an equivalent channel vector between $\mathrm{BS}_{i}$ and $\mathrm{RS}_{i}$.

The signals received at $\mathrm{SS}_{i}$ through CoMP AF relaying from $\mathrm{RS}_{j}, \forall j$, can be written as

$$
y_{r, s_{i}}=\mathbf{h}_{r_{i}, s_{i}}^{H} \mathbf{W}_{r_{i}} \mathbf{y}_{r_{i}}+\sum_{j=1, j \neq i}^{N} \mathbf{h}_{r_{j}, s_{i}}^{H} \mathbf{W}_{r_{j}} \mathbf{y}_{r_{j}}+n_{s_{i}}
$$


where $\mathbf{W}_{r_{i}} \in \mathbb{C}^{N \times N}$ are the BF matrices for $\mathrm{RS}_{i}$ in $\mathrm{C}_{i}$ and $\mathbf{h}_{r_{j}, s_{i}} \in \mathbb{C}^{N \times 1}$ are the channel vectors from $\mathrm{RS}_{j}$ to $\mathrm{SS}_{i}$. Again all entries in $\mathbf{h}_{r_{j}, s_{i}}$ are modeled as flat Rayleigh faded, and the noise $n_{s_{i}}$ are $\sim \mathcal{C N}\left(0, \sigma^{2}\right)$. The total average transmit power from $\mathrm{RS}_{i}, \forall i$, is given by

$$
\begin{aligned}
P_{r} & \triangleq \sum_{i=1}^{N} E\left\{\left\|\mathbf{W}_{r_{i}} \mathbf{y}_{r_{i}}\right\|^{2}\right\} \\
& =\sum_{i=1}^{N}\left(\left\|\mathbf{W}_{r_{i}} \mathbf{h}_{b_{i}, r_{i}}\right\|^{2}+\sigma^{2} \operatorname{tr}\left\{\mathbf{W}_{r_{i}}^{H} \mathbf{W}_{r_{i}}\right\}\right) .
\end{aligned}
$$

For signals sent from $\mathrm{RS}_{i}$, the SINR observed at $\mathrm{SS}_{i}$ can thus be expressed as

$$
\frac{\operatorname{SINR}_{i}^{(r)}=}{\sum_{j=1, j \neq i}^{N}\left|\mathbf{h}_{r_{j}, s_{i}}^{H} \mathbf{W}_{r_{j}, s_{i}} \mathbf{h}_{b_{j}, r_{j}}\right|^{2}+\left.\sigma_{r_{i}} \mathbf{h}_{b_{i}, r_{i}}\right|^{2} \sum_{j=1}^{N}\left\|\mathbf{h}_{r_{j}, s_{i}}^{H} \mathbf{W}_{r_{j}}\right\|^{2}+\sigma^{2}} .
$$

Based on the above system setting and signal models, we study in the sequel BFer designs for CoMP AF relaying in order to maintain the SINR of $\mathrm{SS}_{i}, \forall i$, in retransmissions. We note that the BS BF vectors $\mathbf{w}_{b_{j}}$ are assumed given when designing $\mathbf{W}_{r_{j}}$ since RSs are considered only used in ARQs. Existing results for the design of $\mathbf{w}_{b_{j}}$ can be found for instance in [12], [14], [15], we therefore focus on the design of $\mathbf{W}_{r_{j}}$ for the rest of the paper.

\section{B. Beamformer Design Criteria}

To maintain the $\operatorname{SINR}_{i}^{(r)}, \forall i$, without the loss of generality (WLOG), $\mathbf{W}_{r_{i}}$ can be jointly designed to meet a target SINR, $\gamma_{0}$, according to an SINR constrained power minimization criterion for CoMP AF relaying (SCPM-R), formulated as

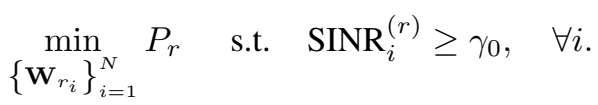

The design criterion of SCPM-R requires the full CSIs for $\mathbf{h}_{b_{i}, r_{i}}$ and $\mathbf{h}_{r_{j}, s_{i}}, \forall i, j$. To cope with the uncertainties in CSI feedbacks, we study robust designs to maintain the SINR for all SSs. To this end, the channel uncertainties are modeled as

$$
\begin{aligned}
\mathbf{h}_{b_{i}, r_{i}} & \triangleq \overline{\mathbf{h}}_{b_{i}, r_{i}}+\mathbf{e}_{b_{i}, r_{i}}, \quad \forall i \\
\mathbf{h}_{r_{j}, s_{i}} & \triangleq \overline{\mathbf{h}}_{r_{j}, s_{i}}+\mathbf{e}_{r_{j}, s_{i}}, \quad \forall i, j
\end{aligned}
$$

where $\overline{\mathbf{h}}_{b_{i}, r_{i}}$ and $\overline{\mathbf{h}}_{r_{j}, s_{i}}$ are the channel vectors available at the $\mathrm{CU}$, and $\mathbf{e}_{b_{i}, r_{i}}$ and $\mathbf{e}_{r_{j}, s_{i}}$, both $\in \mathbb{C}^{N \times 1}$, are their associated unknown uncertainty vectors.

Observe from (3) that the objective function, $P_{r}$, is directly affected by $\mathbf{h}_{b_{i}, r_{i}}$ and, thus, by $\mathbf{e}_{b_{i}, r_{i}}$ as well, which makes $P_{r}$ vary even if $\mathbf{W}_{r_{i}}$ are fixed. Therefore, it becomes more reasonable to find $\mathbf{W}_{r_{i}}$ that minimize the maximum $P_{r}$ for all $\mathbf{e}_{b_{i}, r_{i}}$ fell within a bounded region. According to the models of (6) and (7), the original SCPM-R criterion for $\mathbf{W}_{r_{i}}$ is modified into a robust SCPM criterion for CoMP AF relaying (R-SCPM-R), given by

$$
\begin{array}{lll} 
& \min _{0} p_{0} \\
& p_{0},\left\{\mathbf{W}_{r_{i}}\right\}_{i=1}^{N} \\
\text { s.t. } & P_{r} \leq p_{0} \quad \text { and } \quad \operatorname{SINR}_{i}^{(r)} \geq \gamma_{0}, \quad \forall i \\
& \forall\left\|\mathbf{e}_{r_{j}, s_{i}}\right\| \leq \epsilon_{r, s}, \quad\left\|\mathbf{e}_{b_{i}, r_{i}}\right\| \leq \epsilon_{b, r}, \quad \forall i, j
\end{array}
$$

where $\mathbf{h}_{b_{i}, r_{i}}$ and $\mathbf{h}_{r_{j}, s_{i}}$ in $P_{r}$ and $\operatorname{SINR}_{i}^{(r)}$ are modeled by (6) and (7), respectively.
The design criterion of R-SCPM-R turns out to be a challenging optimization problem, and no optimal solution is obtained in this paper. To resolve the difficulty and approach the final goal, we alternatively consider a degenerated version of R-SCPM-R that only accounts for the uncertainties in $\mathbf{h}_{r_{j}, s_{i}}$. This design criterion is referred to as the semi-robust $\mathrm{SCPM}$ criterion for CoMP AF relaying (SR-SCPM-R), and is formulated as

$$
\min _{\left\{\mathbf{w}_{r_{i}}\right\}_{i=1}^{N}} P_{r} \quad \text { s.t. } \quad \operatorname{SINR}_{i}^{(r)} \geq \gamma_{0}, \forall\left\|\mathbf{e}_{r_{j}, s_{i}}\right\| \leq \epsilon_{r, s}, \forall i, j .
$$

Though less realistic, this design criterion is valid in cases where RSs are allocated enough resources for CSI feedbacks. Given that RSs are closer to their associated BSs than SSs, and are located in places that often have clear line-of-sight transmission paths to their BSs, this assumption seems reasonable because of the high signal quality between BSs and RSs. More importantly, the results obtained from solving this problem can be extended to handle the robust criterion of RSCPM-R, even if in a rather conservative manner. In the next section, we first introduce the BFer designs according to the criterion of SR-SCPM-R (9). We note that the designs of nonrobust $\mathbf{w}_{b_{j}}$ for BSs follow the criterion of (5) but using the SINR expression for downlink CoMP transmission [12], [14], [15]. And the robust designs of $\mathbf{w}_{b_{j}}$ also follow the criterion of (9), with $\mathbf{e}_{r_{j}, s_{i}}$ replaced by $\mathbf{e}_{b_{j}, s_{i}}$.

\section{Semi-Robust BeAmformer Design for CoMP AF RELAYING}

Before we introduce the design methods, we first establish the necessary condition for the optimal BFers, $\mathbf{W}_{r_{i}}$, with which the complexities of designs can be greatly reduced.

Lemma 1: The optimal CoMP AF BF matrices $\mathbf{W}_{r_{i}}, \forall i$, for the criteria of SCPM-R (5) and SR-SCPM-R (9) are rank-one and can always be factorized as

$$
\mathbf{W}_{r_{i}}=\mathbf{w}_{i} \mathbf{h}_{b_{i}, r_{i}}^{H}
$$

where $\mathbf{w}_{i} \in \mathbb{C}^{N \times 1}$.

The proofs of the two problems are similar, and, in fact, the criterion of SCPM-R is a special case of the SR-SCPM-R. We, thus, present the proof for the SR-SCPM-R only.

Proof: The basic idea follows the approaches of [26], [27] to transform the infinite number of constraints from $\forall\left\|\mathbf{e}_{r_{j}, s_{i}}\right\| \leq \epsilon_{r, s}, \forall i, j$, in this case, into a finite number of linear matrix inequalities (LMI) with the S-procedure [20]. The S-procedure states that for $\mathbf{A}, \mathbf{C} \in \mathbb{C}^{N \times N}, \mathbf{b}, \mathbf{v} \in \mathbb{C}^{N \times 1}$ and $c \in \mathbb{R}$, the inequalities $\mathbf{v}^{H} \mathbf{A} \mathbf{v}+\mathbf{b}^{H} \mathbf{v}+\mathbf{v}^{H} \mathbf{b}+c \geq 0$, $\forall \mathbf{v}^{H} \mathbf{C v} \leq 1$, holds if and only if $\exists \lambda \geq 0$ such that

$$
\left[\begin{array}{cc}
\mathbf{A}+\lambda \mathbf{C} & \mathbf{b} \\
\mathbf{b}^{H} & c-\lambda
\end{array}\right] \succeq 0 .
$$

Now if we introduce some auxiliary variables $t_{j, i} \geq 0$, $\forall i, j$, then the constraint of $\operatorname{SINR}_{i}^{(r)} \geq \gamma_{0}$ on (4) can be transformed into three types of constrains, which are, respectively, $\left|\mathbf{h}_{r_{i}, s_{i}}^{H} \mathbf{W}_{r_{i}} \mathbf{h}_{b_{i}, r_{i}}\right|^{2}-\gamma_{0} \sigma^{2}\left\|\mathbf{h}_{r_{i}, s_{i}}^{H} \mathbf{W}_{r_{i}}\right\|^{2} \geq t_{i, i}$, $\left|\mathbf{h}_{r_{j}, s_{i}}^{H} \mathbf{W}_{r_{j}} \mathbf{h}_{b_{j}, r_{j}}\right|^{2}+\sigma^{2}\left\|\mathbf{h}_{r_{j}, s_{i}}^{H} \mathbf{W}_{r_{j}}\right\|^{2} \leq t_{j, i}, j \neq i$, and $t_{i, i} \geq \gamma_{0} \sigma^{2}+\gamma_{0} \sum_{j=1, j \neq i}^{N} t_{j, i}$. Since $\mathbf{h}_{r_{j}, s_{i}}=\overline{\mathbf{h}}_{r_{j}, s_{i}}+\mathbf{e}_{r_{j}, s_{i}}$, 
$\forall\left\|\mathbf{e}_{r_{j}, s_{i}}\right\|^{2} \leq \epsilon_{r, s}^{2}, \forall i, j$, the first two constrains can be transformed with the above S-procedure (11), leading to:

$$
\begin{aligned}
& \min _{\left\{\mathbf{W}_{r_{i}}, t_{j, i}, \lambda_{j, i}\right\}_{i, j=1}^{N}} \sum_{i=1}^{N}\left(\left\|\mathbf{W}_{r_{i}} \mathbf{h}_{b_{i}, r_{i}}\right\|^{2}+\sigma^{2} \operatorname{tr}\left\{\mathbf{W}_{r_{i}}^{H} \mathbf{W}_{r_{i}}\right\}\right) \\
& \text { s.t. } \quad \mathbb{G}_{i, i} \triangleq \text { Blkdiag }\left\{\frac{\lambda_{i, i}}{\epsilon_{r, s}^{2}} \mathbf{I}_{N},-\left(t_{i, i}+\lambda_{i, i}\right)\right\} \\
& +\overline{\mathbf{H}}_{r_{i}, s_{i}}^{H} \mathbb{L}_{i} \overline{\mathbf{H}}_{r_{i}, s_{i}} \succeq 0, \forall i \\
& \mathbb{G}_{j, i} \triangleq \operatorname{Blkdiag}\left\{\frac{\lambda_{j, i}}{\epsilon_{r, s}^{2}} \mathbf{I}_{N}, t_{j, i}-\lambda_{j, i}\right\} \\
& -\overline{\mathbf{H}}_{r_{j}, s_{i}}^{H} \mathbb{Z}_{j, i} \overline{\mathbf{H}}_{r_{j}, s_{i}} \succeq 0, \forall j \neq i \\
& \sum_{j=1, j \neq i}^{N} \gamma_{0} t_{j, i} \leq t_{i, i}-\gamma_{0} \sigma^{2}, \lambda_{j, i}, t_{j, i} \geq 0, \forall i, j
\end{aligned}
$$

where $\mathbb{L}_{i} \triangleq\left(\mathbf{W}_{r_{i}} \mathbf{h}_{b_{i}, r_{i}}\right)\left(\mathbf{W}_{r_{i}} \mathbf{h}_{b_{i}, r_{i}}\right)^{H}-\gamma_{0} \sigma^{2} \mathbf{W}_{r_{i}} \mathbf{W}_{r_{i}}^{H}$, $\mathbb{Z}_{j, i} \triangleq\left(\mathbf{W}_{r_{j}} \mathbf{h}_{b_{j}, r_{j}}\right)\left(\mathbf{W}_{r_{j}} \mathbf{h}_{b_{j}, r_{j}}\right)^{H}+\sigma^{2} \mathbf{W}_{r_{j}} \mathbf{W}_{r_{j}}^{H}$ and $\overline{\mathbf{H}}_{r_{j}, s_{i}} \triangleq\left[\mathbf{I}_{N}, \overline{\mathbf{h}}_{r_{j}, s_{i}}\right], \forall i, j$. The function of Blkdiag $\{\mathbf{A}, \mathbf{B}\}$ constructs a block diagonal matrix out of the square matrices $\mathbf{A}$ and $\mathbf{B}$.

The Lagrangian for (12) can be shown to be

$$
\begin{aligned}
L=P_{r}-\sum_{i, j=1}^{N} \operatorname{tr}\left\{\mathbf{Y}_{j, i} \mathbb{G}_{j, i}\right\} & \\
& -\sum_{i=1}^{N} \rho_{i}\left(t_{i, i}-\gamma_{0} \sigma^{2}-\sum_{j=1, j \neq i}^{N} \gamma_{0} t_{j, i}\right)
\end{aligned}
$$

where $P_{r}$ is defined in (3), and $\rho_{i} \geq 0$ and $\mathbf{Y}_{j, i} \succeq 0$ are Lagrangian multipliers. Taking the derivative of (13) with respect to (w.r.t.) $\mathbf{W}_{r_{i}}$ and setting it to the zero matrix yields

$$
\mathbf{W}_{r_{i}}=\left(\sigma^{2} \boldsymbol{\Omega}_{i}\right)^{-1} \boldsymbol{\Omega}_{i}^{\prime} \mathbf{W}_{r_{i}} \mathbf{h}_{b_{i}, r_{i}} \mathbf{h}_{b_{i}, r_{i}}^{H}
$$

with $\boldsymbol{\Omega}_{i}^{\prime} \triangleq \overline{\mathbf{H}}_{r_{i}, s_{i}} \mathbf{Y}_{i, i} \overline{\mathbf{H}}_{r_{i}, s_{i}}^{H}-\sum_{m=1, m \neq i}^{N} \overline{\mathbf{H}}_{r_{i}, s_{m}} \mathbf{Y}_{i, m} \overline{\mathbf{H}}_{r_{i}, s_{m}}^{H}-\mathbf{I}$ and $\boldsymbol{\Omega}_{i} \triangleq \gamma_{0} \overline{\mathbf{H}}_{r_{i}, s_{i}} \mathbf{Y}_{i, i} \overline{\mathbf{H}}_{r_{i}, s_{i}}^{H}+\sum_{m=1, m \neq i}^{N} \overline{\mathbf{H}}_{r_{i}, s_{m}} \mathbf{Y}_{i, m} \overline{\mathbf{H}}_{r_{i}, s_{m}}^{H}+$ $\mathbf{I} \succeq 0$ being of full rank. This completes the proof.

Lemma 1 implies that, WLOG, the AF BF matrices can be expressed as $\mathbf{W}_{r_{i}}=\mathbf{w}_{r_{i}} \mathbf{h}_{b_{i}, r_{i}}^{H} /\left\|\mathbf{h}_{b_{i}, r_{i}}\right\|$, where $\mathbf{w}_{r_{i}} \in \mathbb{C}^{N \times 1}$ and $\mathbf{h}_{b_{i}, r_{i}} \triangleq \mathbf{H}_{b_{i}, r_{i}} \mathbf{w}_{b_{i}}$. Supposed that $\mathbf{w}_{b_{i}}$ are made available for $\mathrm{RS}_{i}$ in ARQ, only $\mathbf{h}_{b_{i}, r_{i}}$ are needed to be fed back to the CU. The CU, in this case, only needs to inform $\mathrm{RS}_{i}$ of $\mathbf{w}_{r_{i}}$ as well. The total transmit power of relays, (3), becomes $P_{r}=\sum_{i=1}^{N} c_{i}\left\|\mathbf{w}_{r_{i}}\right\|^{2}$ with $c_{i} \triangleq\left(\left\|\mathbf{h}_{b_{i}, r_{i}}\right\|^{2}+\sigma^{2}\right)$. Supposed that no uncertainty occurs in the feedbacks of $\mathbf{h}_{r_{j}, s_{i}}, \forall i, j$, the SCPM-R criterion of (5) can then be reformulated as

$$
\begin{array}{ll} 
& \min p_{0},\left\{\mathbf{w}_{r_{i}}\right\}_{i=1}^{N} \\
\text { s.t. } & \sum_{i=1}^{N} c_{i}\left\|\mathbf{w}_{r_{i}}\right\|^{2} \leq p_{0}, \\
& \frac{d_{i}}{\gamma_{0}}\left|\mathbf{h}_{r_{i}, s_{i}}^{H} \mathbf{w}_{r_{i}}\right|^{2} \geq \sum_{j=1, j \neq i}^{N} c_{j}\left|\mathbf{h}_{r_{j}, s_{i}}^{H} \mathbf{w}_{r_{j}}\right|^{2}+\sigma^{2}, \forall i
\end{array}
$$

where $d_{i} \triangleq\left(\left\|\mathbf{h}_{b_{i}, r_{i}}\right\|^{2}-\gamma_{0} \sigma^{2}\right)$. This formulation is a standard form of QCQP in [28]. The optimal BF vectors $\mathbf{w}_{r_{i}}$ can thus be solved directly with the algorithms presented therein.
A. Semi-Robust Beamformer Design Based on the SProcedure and SDR

Based on the form of $\mathbf{W}_{r_{i}}=\mathbf{w}_{r_{i}} \mathbf{h}_{b_{i}, r_{i}}^{H} /\left\|\mathbf{h}_{b_{i}, r_{i}}\right\|$, we may define $\alpha_{j, i} \triangleq\left|\left(\overline{\mathbf{h}}_{r_{j}, s_{i}}+\mathbf{e}_{r_{j}, s_{i}}\right)^{H} \mathbf{w}_{r_{j}}\right|^{2}$ such that the SINR expression of (4) under the uncertainties of $\mathbf{e}_{r_{j}, s_{i}}$ in (7) can be rewritten as

$$
\operatorname{SINR}_{i}^{(r)}=\frac{\left\|\mathbf{h}_{b_{i}, r_{i}}\right\|^{2} \alpha_{i, i}}{\sum_{j=1, j \neq i}^{N}\left\|\mathbf{h}_{b_{j}, r_{j}}\right\|^{2} \alpha_{j, i}+\sigma^{2} \sum_{j=1}^{N} \alpha_{j, i}+\sigma^{2}} .
$$

The SR-SCPM-R criterion of (9) ensures that every SS will be served with an SINR at least better than the target value, $\gamma_{0}$, for all channel uncertainties, $\mathbf{e}_{r_{j}, s_{i}}$, whose $\left\|\mathbf{e}_{r_{j}, s_{i}}\right\| \leq \epsilon_{r, s}$. This, in fact, imposes an infinite number of constraints on the SR-SCPM-R problem. As pointed out in Lemma 1, the infinite number of constraints can be reduced to a finite number of LMIs if we first introduce some auxiliary variables $t_{j, i} \geq 0$, $\forall i, j$, to transform the constraint of $\operatorname{SINR}_{i}^{(r)} \geq \gamma_{0}$ on (16) into three ones which are $\left\|\mathbf{h}_{b_{i}, r_{i}}\right\|^{2} \alpha_{i, i}-\gamma_{0} \sigma^{2} \alpha_{i, i} \geq \gamma_{0} t_{i, i}$, $\left\|\mathbf{h}_{b_{j}, r_{j}}\right\|^{2} \alpha_{j, i}+\sigma^{2} \alpha_{j, i} \leq t_{j, i}, j \neq i, \forall\left\|\mathbf{e}_{r_{j}, s_{i}}\right\|^{2} \leq \epsilon_{r, s}^{2}$, $\mathbf{e}_{r_{j}, s_{i}} \in \mathbb{C}^{N \times 1}$, and $t_{i, i} \geq \sigma^{2}+\sum_{j=1, j \neq i}^{N} t_{j, i}$. The constrains that involve $\mathbf{e}_{r_{j}, s_{i}}$, namely $\alpha_{j, i}$, can then be transformed with the S-procedure according to (11) since $\alpha_{j, i}$ are quadratic functions in $\mathbf{e}_{r_{j}, s_{i}}$. Consequently, by the definitions of $d_{i} \triangleq\left\|\mathbf{h}_{b_{i}, r_{i}}\right\|^{2}-\gamma_{0} \sigma^{2}, c_{i} \triangleq\left\|\mathbf{h}_{b_{i}, r_{i}}\right\|^{2}+\sigma^{2}$ and $P_{r}=$ $\sum_{i=1}^{N} c_{i}\left\|\mathbf{w}_{r_{i}}\right\|^{2} \triangleq \sum_{i=1}^{N} \operatorname{tr}\left\{\mathbb{W}_{r_{i}}\right\}$ with $\mathbb{W}_{r_{i}} \triangleq c_{i} \mathbf{w}_{r_{i}} \mathbf{w}_{r_{i}}^{H}$, the SR-SCMP-R criterion can be reformulated into

$$
\begin{aligned}
& \min _{\left\{\mathbb{W}_{r_{i}}, t_{j, i}, \lambda_{j, i}\right\}_{i, j=1}^{N}} \sum_{i=1}^{N} \operatorname{tr}\left\{\mathbb{W}_{r_{i}}\right\} \\
& \text { s.t. } \operatorname{Blkdiag}\left\{\frac{\lambda_{i, i}}{\epsilon_{r, s}^{2}} \mathbf{I}_{N},-\left(t_{i, i}+\lambda_{i, i}\right)\right\} \\
& +\frac{d_{i}}{c_{i} \gamma_{0}} \overline{\mathbf{H}}_{r_{i}, s_{i}}^{H} \mathbb{W}_{r_{i}} \overline{\mathbf{H}}_{r_{i}, s_{i}} \succeq 0, \quad \forall i \\
& \text { Blkdiag }\left\{\frac{\lambda_{j, i}}{\epsilon_{r, s}^{2}} \mathbf{I}_{N}, t_{j, i}-\lambda_{j, i}\right\} \\
& -\overline{\mathbf{H}}_{r_{j}, s_{i}}^{H} \mathbb{W}_{r_{j}} \mathbf{H}_{r_{j}, s_{i}} \succeq 0, \quad \forall j \neq i \\
& \sum_{j=1, j \neq i}^{N} t_{j, i}+\sigma^{2} \leq t_{i, i}, \lambda_{j, i}, t_{j, i} \geq 0, \quad \forall i, j \\
& \operatorname{rank}\left\{\mathbb{W}_{r_{i}}\right\}=1, \quad \mathbb{W}_{r_{i}} \succeq 0,
\end{aligned}
$$

where $\overline{\mathbf{H}}_{r_{j}, s_{i}} \triangleq\left[\mathbf{I}_{N} \overline{\mathbf{h}}_{r_{j}, s_{i}}\right], \forall i, j$. If we relax the constraint of $\operatorname{rank}\left\{\mathbb{W}_{r_{i}}\right\}=1$, then the programming problem becomes a standard form of SDP. The corresponding BFers, $\mathbb{W}_{r_{i}}$, can be obtained with the available optimization solvers such as SeDuMe or CVX [22], [23].

Based on the complexity analysis on the interior-point method for solving SDP in [29], the complexity order for solving (17) can be shown to be $\mathcal{O}\left(N^{6}\right)$ per iteration ${ }^{1}$. The BFers $\mathbb{W}_{r_{i}}$ are optimal if they satisfy the rank-one condition, too. By inspection from our simulations, $\mathbb{W}_{r_{i}}$ seem to always satisfy the rank-one condition, though this phenomenon is not theoretically proven herein. Similar observations had been reported in [26], [27] as well for other robust designs using the S-procedure. The proposed SDR approach (17) can thus be practically considered as a nearly optimal solver.

${ }^{1}$ According to the method of [29], the number of iterations is proportional to $\mathcal{O}\left(N^{0.5}\right)$. However, due to space limitation, we do not study the number of iterations for the two-tier QCQP method (21) to be presented in the next section. The entire scheme is considered infeasible in simulations once the number of iterations exceeds 100 . We, therefore, compare the two algorithms' complexities on a per-iteration basis only. 


\section{B. Semi-Robust Beamformer Design Based on a Two-Tier Iterative $Q C Q P$}

The SDR approach is, however, less friendly for practical implementations. Motivated by the results in [30], [31], we present next a more intuitive way to transform the infinite number of constraints in the SR-SCMP-R criterion of (9). The approach is less complex and can also be extended to handle the R-SCPM-R problem of (8). To resolve the conservativeness often seen in this approach, we introduce a number of auxiliary variables and inequalities to ensure the tightness in the process of constraint transformation. Together with a new formulation for programming, this leads to an iterative but nearly closed-form solver. The iterative solver presents a performance very close to the SDR method for (17), and provides us an insight into the structure of robust BFers for CoMP AF relaying.

Recall the formulation of (9). To relieve the infinite number of constraints in programming, we need to bound the effects of $\mathbf{e}_{r_{j}, s_{i}}$ in $\alpha_{i, i}$ and $\alpha_{j, i}$ of (16), $\forall\left\|\mathbf{e}_{r_{j}, s_{i}}\right\| \leq \epsilon_{r, s}, \forall i, j$. Observing from the form of $\alpha_{j, i}$ in (16), $\mathbf{e}_{r_{j}, s_{i}}$ are embedded in $\mathbf{e}_{r_{j}, s_{i}}^{H} \mathbf{w}_{r_{j}}$ whose 2-norms can be upper bounded by $\left|\mathbf{e}_{r_{j}, s_{i}}^{H} \mathbf{w}_{r_{j}}\right| \leq\left\|\mathbf{e}_{r_{j}, s_{i}}\right\|\left\|\mathbf{w}_{r_{j}}\right\| \leq \epsilon_{r, s}\left\|\mathbf{w}_{r_{j}}\right\|, \forall i, j$. Substituting this upper bound into $\alpha_{i, i}$ followed by some mathematical manipulations, it results in

$$
\begin{aligned}
\alpha_{i, i}= & \left|\left(\overline{\mathbf{h}}_{r_{i}, s_{i}}+\mathbf{e}_{r_{i}, s_{i}}\right)^{H} \mathbf{w}_{r_{i}}\right|^{2} \\
\geq & \left(\left|\overline{\mathbf{h}}_{r_{i}, s_{i}}^{H} \mathbf{w}_{r_{i}}\right|-\left|\mathbf{e}_{r_{i}, s_{i}}^{H} \mathbf{w}_{r_{i}}\right|\right)^{2} \\
\geq & \left|\overline{\mathbf{h}}_{r_{i}, s_{i}}^{H} \mathbf{w}_{r_{i}}\right|^{2} \\
& \quad-2 \epsilon_{r, s}\left|\overline{\mathbf{h}}_{r_{i}, s_{i}}^{H} \mathbf{w}_{r_{i}}\right||| \mathbf{w}_{r_{i}}\left\|+\epsilon_{r, s}^{2}\right\| \mathbf{w}_{r_{i}} \|^{2}
\end{aligned}
$$

if $\left|\overline{\mathbf{h}}_{r_{i}, s_{i}}^{H} \mathbf{w}_{r_{i}}\right| \geq \epsilon_{r, s}\left\|\mathbf{w}_{r_{i}}\right\|$. The lower bound is, in fact, tight for the SR-SCMP-R problem of (9) as there exist $\mathbf{e}_{r_{i}, s_{i}}$ that make the equality hold in the above expression.

This lower bound can further be expressed in a quadratic form if we define some parameters $v_{i, i}, \forall i$, such that $\left|\overline{\mathbf{h}}_{r_{i}, s_{i}}^{H} \mathbf{w}_{r_{i}}^{H}\right| \leq v_{i, i}\left\|\overline{\mathbf{h}}_{r_{i}, s_{i}}\right\|\left\|\mathbf{w}_{r_{i}}\right\|$. Specifically, we have

$$
\alpha_{i, i} \geq \mathbf{w}_{r_{i}}^{H}\left[\overline{\mathbf{h}}_{r_{i}, s_{i}} \overline{\mathbf{h}}_{r_{i}, s_{i}}^{H}+\left(\epsilon_{r, s}^{2}-2 \epsilon_{r, s} v_{i, i}\left\|\overline{\mathbf{h}}_{r_{i}, s_{i}}\right\|\right) \mathbf{I}_{N}\right] \mathbf{w}_{r_{i}} .
$$

To satisfy the condition of $\left|\overline{\mathbf{h}}_{r_{i}, s_{i}}^{H} \mathbf{w}_{r_{i}}\right| \geq \epsilon_{r, s}\left\|\mathbf{w}_{r_{i}}\right\|, v_{i, i}$ must also satisfy $v_{i, i} \geq \frac{\epsilon_{r, s}}{\left\|\mathbf{h}_{r_{i}, s_{i}}\right\|}$.

Similarly, define $v_{j, i}, \forall j \neq i$, such that $\left|\overline{\mathbf{h}}_{r_{j}, s_{i}}^{H} \mathbf{w}_{r_{j}}^{H}\right| \leq$ $v_{j, i}\left\|\overline{\mathbf{h}}_{r_{j}, s_{i}}\right\|\left\|\mathbf{w}_{r_{j}}\right\|$, we can also have

$$
\begin{aligned}
\alpha_{j, i} \leq & \left|\left(\overline{\mathbf{h}}_{r_{j}, s_{i}}+\mathbf{e}_{r_{j}, s_{i}}\right)^{H} \mathbf{w}_{r_{j}}\right|^{2} \\
\leq & \left(\left|\overline{\mathbf{h}}_{r_{j}, s_{i}} \mathbf{w}_{r_{j}}\right|+\epsilon_{r, s}\left\|\mathbf{w}_{r_{j}}\right\|\right)^{2} \\
\leq & \mathbf{w}_{r_{j}}^{H}\left[\overline{\mathbf{h}}_{r_{j}, s_{i}} \overline{\mathbf{h}}_{r_{j}, s_{i}}^{H}\right. \\
& \left.\quad+\left(\epsilon_{r, s}^{2}+2 \epsilon_{r, s} v_{j, i}\left\|\overline{\mathbf{h}}_{r_{j}, s_{i}}\right\|\right) \mathbf{I}_{N}\right] \mathbf{w}_{r_{j}} .
\end{aligned}
$$

Again, the worst $\mathbf{e}_{r_{j}, s_{i}}$ will also make the equality hold for the first inequality above.

Based on (19) and (20), the SR-SCMP-R criterion of (9)
Algorithm 1 A two-tier iterative QCQP scheme for the SRSCPM-R problem.

1: Initialize $n=0$ and set $v_{i, i}^{(0)}=1, \forall i$, and $v_{j, i}^{(0)}=0.001$, $\forall j \neq i$.

2: Define $\mathbf{G}_{j, i} \triangleq v_{j, i}^{2}\left\|\overline{\mathbf{h}}_{r_{j}, s_{i}}\right\|^{2} \mathbf{I}_{N}-\overline{\mathbf{h}}_{r_{j}, s_{i}} \overline{\mathbf{h}}_{r_{i}, s_{i}}^{H}, \forall i, j$.

3: Define $\boldsymbol{\Psi}_{i} \triangleq c_{i} \mathbf{I}_{N}-\frac{\lambda_{i}}{\gamma_{0}} \mathbf{A}_{i}+\sum_{j=1, j \neq i}^{N} \lambda_{j} \mathbf{B}_{i, j}-$ $\sum_{j=1}^{N} \tau_{i, j} \mathbf{G}_{i, j}, \forall i$.

4: Define $\boldsymbol{\Phi}_{i, i} \triangleq \boldsymbol{\Psi}_{i}+\frac{\lambda_{i} d_{i}}{\gamma_{0}}\left(\overline{\mathbf{h}}_{r_{i}, s_{i}} \overline{\mathbf{h}}_{r_{i}, s_{i}}^{H}\right)$ and $\boldsymbol{\Phi}_{j, i} \triangleq \boldsymbol{\Psi}_{j}-$ $\lambda_{i} c_{j} \overline{\mathbf{h}}_{r_{j}, s_{i}} \overline{\mathbf{h}}_{r_{j}, s_{i}}^{H}, \forall j \neq i$.

5: while $\left|v_{j, i}^{(n+1)}-v_{j, i}^{(n)}\right|>0, \forall i, j$ do

6: $\quad$ Initialize $m=0$ and set $\lambda_{i}(0)=0$

7: $\quad$ while $\left|\lambda_{i}(m+1)-\lambda_{i}(m)\right|>0, \forall i$. do

8: $\quad$ Evaluate $\boldsymbol{\Phi}_{i, i}(m)$ with $\lambda_{i}=\lambda_{i}(m), \tau_{j, i}=\tau_{j, i}(m)$ and $v_{j, i}=v_{j, i}^{(n)}$ in $\boldsymbol{\Phi}_{i, i}$, and compute

$$
\begin{aligned}
\lambda_{i}(m+1) & =\frac{\gamma_{0}}{d_{i} \overline{\mathbf{h}}_{r_{i}, s_{i}}^{H}\left(\boldsymbol{\Phi}_{i, i}(m)\right)^{-1} \overline{\mathbf{h}}_{r_{i}, s_{i}}}, & & \forall i \\
\tau_{i, i}(m+1) & =\frac{\epsilon_{r, s} d_{i} \lambda_{i}(m)}{\gamma_{0} v_{i, i}^{(n)}\left\|\overline{\mathbf{h}}_{r_{i}, s_{i}}\right\|}, & & \forall i \\
\tau_{j, i}(m+1) & =\frac{\epsilon_{r, s} c_{j} \lambda_{i}(m)}{v_{j, i}^{(n)}\left\|\overline{\mathbf{h}}_{r_{j}, s_{i}}\right\|}, & & \forall j \neq i .
\end{aligned}
$$

9: $\quad$ Set $m=m+1$

\section{0: end while}

11: $\quad$ Evaluate $\boldsymbol{\Phi}_{j, i}^{(n)}$ with $\lambda_{i}=\lambda_{i}(m), \tau_{j, i}=\tau_{j, i}(m)$ and $v_{j, i}=v_{j, i}^{(n)}$ in $\boldsymbol{\Phi}_{j, i}$, and then compute

$$
\begin{array}{ll}
v_{i, i}^{(n+1)}=\frac{\gamma_{0}}{\lambda_{i}(m) d_{i}\left\|\overline{\mathbf{h}}_{r_{i}, s_{i}}\right\|\left\|\left(\boldsymbol{\Phi}_{i, i}^{(n)}\right)^{-1} \overline{\mathbf{h}}_{r_{i}, s_{i}}\right\|}, & \forall i \\
v_{j, i}^{(n+1)}=\frac{1}{\lambda_{i}(m) c_{j}\left\|\overline{\mathbf{h}}_{r_{j}, s_{i}}\right\|\left\|\left(\boldsymbol{\Phi}_{j, i}^{(n)}\right)^{-1} \overline{\mathbf{h}}_{r_{j}, s_{i}}\right\|}, \quad \forall j \neq i .
\end{array}
$$

12: $\quad$ Set $n=n+1$

\section{3: end while}

14: if The iterations above converge then

15: Evaluate

$$
\begin{aligned}
{[\mathbf{F}]_{i, i}=} & \left(\frac{\lambda_{i}(m) d_{i}}{r_{0}}\right)^{2} \times \\
& \overline{\mathbf{h}}_{r_{i}, s_{i}}^{H}\left(\boldsymbol{\Phi}_{i, i}^{(n)}\right)^{-1}\left(\frac{\mathbf{A}_{i}}{r_{0}}\right)\left(\boldsymbol{\Phi}_{i, i}^{(n)}\right)^{-1} \overline{\mathbf{h}}_{r_{i}, s_{i}}, \quad \forall i .
\end{aligned}
$$

16: Evaluate

$$
\begin{aligned}
{[\mathbf{F}]_{i, j}=} & -\left(\frac{\lambda_{j}(m) d_{j}}{r_{0}}\right)^{2} \times \\
& \overline{\mathbf{h}}_{r_{j}, s_{j}}^{H}\left(\boldsymbol{\Phi}_{j, j}^{(n)}\right)^{-1} \mathbf{B}_{j, i}\left(\boldsymbol{\Phi}_{j, j}^{(n)}\right)^{-1} \overline{\mathbf{h}}_{r_{j}, s_{j}}, \quad \forall i \neq j
\end{aligned}
$$

17: Define $\delta_{i} \triangleq \sigma \sqrt{\sum_{j=1}^{N}\left[\mathbf{F}^{-1}\right]_{i, j}}$ and compute

$$
\mathbf{W}_{r_{i}}=\frac{\lambda_{i}(m) d_{i} \delta_{i}}{\gamma_{0}}\left(\boldsymbol{\Phi}_{i, i}^{(n)}\right)^{-1} \overline{\mathbf{h}}_{r_{i}, s_{i}} \mathbf{h}_{b_{i}, r_{i}}^{H}, \forall i .
$$

18: else

19: The pair $\left\{\gamma_{0}, \epsilon_{r, s}\right\}$ is infeasible for the given channel realization. 
can be reformulated as

$$
\begin{array}{lll} 
& \min _{\left\{\mathbf{w}_{r_{i}}, v_{j, i}\right\}_{i, j=1}^{N}} \sum_{i=1}^{N} c_{i}\left\|\mathbf{w}_{r_{i}}\right\|^{2} & \\
\text { s.t. } & \frac{1}{\gamma_{0}} \mathbf{w}_{r_{i}}^{H} \mathbf{A}_{i} \mathbf{w}_{r_{i}} \geq \sum_{j=1, j \neq i}^{N} \mathbf{w}_{r_{j}}^{H} \mathbf{B}_{j, i} \mathbf{w}_{r_{j}}+\sigma^{2}, & \forall i \\
& \left|\overline{\mathbf{h}}_{r_{j}, s_{i}}^{H} \mathbf{w}_{r_{j}}^{H}\right|^{2} \leq v_{j, i}^{2}\left\|\overline{\mathbf{h}}_{r_{j}, s_{i}}\right\|^{2}\left\|\mathbf{w}_{r_{j}}\right\|^{2}, & \forall i, j \\
& v_{i, i} \geq \frac{\epsilon_{r, s}}{\left\|\mathbf{h}_{r_{i}, s_{i}}\right\|}, v_{j, i} \geq 0, & \forall i, j
\end{array}
$$

where $d_{i} \triangleq\left\|\mathbf{h}_{b_{i}, r_{i}}\right\|^{2}-\gamma_{0} \sigma^{2}, \mathbf{A}_{i} \triangleq d_{i}\left[\overline{\mathbf{h}}_{r_{i}, s_{i}} \overline{\mathbf{h}}_{r_{i}, s_{i}}^{H}+\right.$ $\left.\left(\epsilon_{r, s}^{2}-2 \epsilon_{r, s} v_{i, i}\left\|\overline{\mathbf{h}}_{r_{i}, s_{i}}\right\|\right) \mathbf{I}_{N}\right], c_{i} \triangleq\left\|\mathbf{h}_{b_{i}, r_{i}}\right\|^{2}+\sigma^{2}$ and $\mathbf{B}_{j, i} \triangleq$ $c_{j}\left[\overline{\mathbf{h}}_{r_{j}, s_{i}} \overline{\mathbf{h}}_{r_{j}, s_{i}}^{H}+\left(\epsilon_{r, s}^{2}+2 \epsilon_{r, s} v_{j, i}\left\|\overline{\mathbf{h}}_{r_{j}, s_{i}}\right\|\right) \mathbf{I}_{N}\right], \forall j \neq i$. The infinite number of constraints in the original formulation of (9) have been transformed into finite ones in (21). And the tightness of the new constraints can be preserved if one can find the optimal $\hat{v}_{j, i}$ and $\hat{\mathbf{w}}_{r_{j}}$ that satisfy the equalities of $\left|\overline{\mathbf{h}}_{r_{j}, s_{i}}^{H} \mathbf{w}_{r_{j}}\right| \leq v_{j, i}\left\|\overline{\mathbf{h}}_{r_{j}, s_{i}}\right\|\left\|\mathbf{w}_{r_{j}}\right\|, \forall i, j$.

Although, the formulation of (21) is not in a convex form, given $v_{j, i}$, the programming can be viewed as a particular form of QCQP in [28], and will yield the optimal $\hat{\mathbf{w}}_{r_{i}}$ provided that $v_{j, i}$ are optimal, too. The same argument applies to $\hat{v}_{j, i}$ as well given $\hat{\mathbf{w}}_{r_{i}}$. Based on this idea, we develop a two-tier iterative algorithm to find the $v_{j, i}$ and $\mathbf{w}_{r_{i}}$ according to the KarushKuhn-Tucker (KKT) conditions of (21). The expressions of $\mathbf{w}_{r_{i}}$ and $v_{j, i}$ are derived in the appendix followed by a fixed-point iterative scheme to solve their dual variables in Algorithm 1. The algorithm's complexity order is $\mathcal{O}\left(N^{5}\right)$ per iteration. The iterative method in general guarantees that the solutions satisfy the necessary conditions for (21). Nevertheless, simulation results show that the resultant BFers perform almost the same as do the BFers obtained with the SDR approach for (17).

\section{Robust BeAmFormer Design For COMP AF RELAYING}

In this section, we extend the results obtained previously to study robust BFer designs that also take into account the BSto-RS CSI uncertainties. However, Lemma 1 does not apply to the R-SCPM-R criterion of (8). To circumvent this difficulty, we adopt below a rather heuristic approach to analyze the structure of the optimal BFers.

Recall the formulation of (8), the uncertainties $\mathbf{e}_{r_{j}, s_{i}}$ and $\mathbf{e}_{b_{i}, r_{i}}$ in fact impose an infinite number of constrains on programming, hence an infinite number of dual variables too. To resolve this difficulty, we may define an error matrix $\mathcal{E} \triangleq\left[\left\{\mathbf{e}_{r_{j}, s_{i}}\right\}_{i, j=1}^{N},\left\{\mathbf{e}_{b_{i}, r_{i}}\right\}_{i=1}^{N}\right]$ in $\mathbb{C}^{N \times\left(N^{2}+N\right)}$, and accordingly define the dual variables as functions of $\mathcal{E}$ in this case. Replacing the summation of infinite terms in the Lagrangian, $L$, with an integral over an uncertainty ellipsoid defined as $\mathcal{R} \triangleq\left\{\mathcal{E} \mid\left\|\mathbf{e}_{r_{j}, s_{i}}\right\| \leq \epsilon_{r, s},\left\|\mathbf{e}_{b_{i}, r_{i}}\right\| \leq \epsilon_{b, r}, \forall i, j\right\}$, we have (22) where $\tau(\mathcal{E})$ and $\lambda_{i}(\mathcal{E})$ are dual variables, and $\eta_{i, i}=\frac{1}{\gamma_{0}}$ and $\eta_{i, j}=-1, \forall i \neq j$. Take the derivative of (22) w.r.t. $\mathbf{W}_{r_{i}}^{\gamma_{0}}$ and set it to the zero matrix. It is routine to show that

$$
\begin{aligned}
& \mathbf{W}_{r_{i}}=\boldsymbol{\Pi}_{i}\left[\oint_{\mathcal{R}_{i}} \boldsymbol{\Delta}_{i} d \mathcal{E}_{r_{i}}\right] \mathbf{W}_{r_{i}}\left[\oint \mathbf{h}_{b_{i}, r_{i}} \mathbf{h}_{b_{i}, r_{i}}^{H} d \mathbf{e}_{b_{i}, r_{i}}\right] \\
& \stackrel{\epsilon_{b, r} \rightarrow 0}{\approx} \boldsymbol{\Pi}_{i}\left[\oint_{\mathcal{R}_{i}} \boldsymbol{\Delta}_{i} d \mathcal{E}_{r_{i}}\right] \mathbf{W}_{r_{i}} \overline{\mathbf{h}}_{b_{i}, r_{i}} \overline{\mathbf{h}}_{b_{i}, r_{i}}^{H}
\end{aligned}
$$

where $\boldsymbol{\Delta}_{i} \triangleq \frac{\lambda_{i}(\mathcal{E})}{r_{0}} \mathbf{h}_{r_{i}, s_{i}} \mathbf{h}_{r_{i}, s_{i}}^{H}-\sum_{m=1, m \neq i}^{N} \lambda_{m}(\mathcal{E}) \mathbf{h}_{r_{i}, s_{m}} \mathbf{h}_{r_{i}, s_{m}}^{H}-$ $\mathbf{I}_{N}$, and $\boldsymbol{\Pi}_{i}^{-1} \triangleq\left(\sigma^{2} \mathbf{I}_{N}+\sigma^{2} \oint_{\mathcal{R}_{i}} \sum_{m=1}^{N} \lambda_{m}(\mathcal{E}) \mathbf{h}_{r_{i}, s_{m}} \mathbf{h}_{r_{i}, s_{m}}^{H} d \mathcal{E}_{r_{i}}\right)$ with $\mathcal{E}_{r_{i}} \triangleq\left[\left\{\mathbf{e}_{r_{i}, s_{m}}\right\}_{m=1}^{N}\right]$ and $\mathcal{R}_{i} \triangleq\left\{\mathcal{E}_{r_{i}} \mid\left\|\mathbf{e}_{r_{i}, s_{m}}\right\| \leq \epsilon_{r, s}\right.$, $\forall m\}$.

This echoes the rank-one condition in (14), though heuristically. Motivated by this approximation, and to keep the complexity low, we still define the form of the BF matrices as $\mathbf{w}_{r_{i}} \overline{\mathbf{h}}_{b_{i}, r_{i}}^{H} /\left\|\overline{\mathbf{h}}_{b_{i}, r_{i}}\right\| \triangleq \mathbf{w}_{r_{i}} \mathbf{p}_{i}^{H}$, where $\mathbf{w}_{r_{i}} \in \mathbb{C}^{N \times 1}$. This idea is also based on the observation from (10) that RSs first combine their received signals with $\overline{\mathbf{h}}_{b_{i}, r_{i}}$ in a sense to maximize their signal to noise ratios. The combined signals are then normalized and beamformed to their associated SSs to maintain the final SINR at SSs. Under this BFer structure, the CU still informs $\mathrm{RS}_{i}$ of their own $\mathbf{w}_{r_{i}}$ only, and the final SINRs observed by $\mathrm{SS}_{i}$ become

$$
\frac{\operatorname{SINR}_{i}^{(r)}=}{\sum_{j=1, j \neq i}^{N}\left(\left\|\overline{\mathbf{h}}_{b_{j}, r_{j}}\right\|+\mathbf{p}_{j}^{H} \mathbf{e}_{b_{j}, r_{j}}\right)^{2} \alpha_{j, i}+\sigma^{2} \sum_{j=1}^{N} \alpha_{j, i}+\mathbf{p}^{2}}
$$

where $\mathbf{p}_{i}$ are the receive BFers. Clearly, the SINR has a lower bound when its numerator is set to a lower bound, and its denominator set to an upper bound. Following the approach in Section III-B, and using the triangular inequality and the fact that $\left|\mathbf{p}_{j}^{H} \mathbf{e}_{b_{j}, r_{j}}\right| \leq\left\|\mathbf{p}_{j}\right\|\left\|\mathbf{e}_{b_{j}, r_{j}}\right\| \leq \epsilon_{b, r}\left\|\mathbf{p}_{j}\right\|$, it is straightforward to show that (24) is bounded from below by

$$
\begin{aligned}
& \operatorname{SINR}_{i}^{(r, w o r s t)}= \\
& \frac{\left(\left\|\overline{\mathbf{h}}_{b_{i}, r_{i}}\right\|-\epsilon_{b, r}\left\|\mathbf{p}_{i}\right\|\right)^{2} \alpha_{i, i}}{\sum_{j=1, j \neq i}^{N}\left(\left\|\overline{\mathbf{h}}_{b_{j}, r_{j}}\right\|+\epsilon_{b, r}\left\|\mathbf{p}_{j}\right\|\right)^{2} \alpha_{j, i}+\left(\sum_{j=1}^{N} \alpha_{j, i}+1\right) \sigma^{2}} .
\end{aligned}
$$

This lower bound is in general lossy as there exists no $\mathbf{e}_{b_{i}, r_{i}}$ that can minimize the numerator of (24) and, in the meantime, maximizes its denominators, too. However, looking at the bright side, this formulation allows us to apply the results in Section III-B to this more general robust design problem if we reformulate the total power of RSs as

$$
\begin{aligned}
P_{r} & =\sum_{i=1}^{N}\left(\left\|\overline{\mathbf{h}}_{b_{i}, r_{i}}\right\|+\left.\mathbf{p}_{i}^{H} \mathbf{e}_{b_{i}, r_{i}}\right|^{2}+\sigma^{2}\right)\left\|\mathbf{w}_{r_{i}}\right\|^{2} \\
& \leq \sum_{i=1}^{N} \hat{c}_{i}\left\|\mathbf{w}_{r_{i}}\right\|^{2}
\end{aligned}
$$

where $\hat{c}_{i} \triangleq\left(\left\|\overline{\mathbf{h}}_{b_{i}, r_{i}}\right\|+\epsilon_{b, r}\left\|\mathbf{p}_{i}\right\|\right)^{2}+\sigma^{2}$. Consequently, the original R-SCMP-R problem of (8) is approximated by a rather conservative design criterion of the form

$$
\begin{array}{ll} 
& \min \sum_{\left.\mathbf{w}_{r_{i}}\right\}_{i=1}^{N}} \sum_{i=1}^{N} \hat{c}_{i}\left\|\mathbf{w}_{r_{i}}\right\|^{2} \\
\text { s.t. } & \operatorname{SINR}_{i}^{(r, \text { worst })} \geq \gamma_{0}, \forall\left\|\mathbf{e}_{r_{j}, s_{i}}\right\| \leq \epsilon_{r, s}, \forall i, j .
\end{array}
$$

This problem can be solved efficiently with the iterative QCQP solver in Section III-B. More importantly, it allows us to assess the performance degradation due to $\mathbf{e}_{b_{i}, r_{i}}$ in $\mathbf{h}_{b_{i}, r_{i}}$. Simulations results are presented next to compare the various BF designs discussed so far. 


$$
\begin{aligned}
L=p_{0}-\oint_{\mathcal{R}} \tau(\mathcal{E})\left(p_{0}-\sum_{i=1}^{N}\left(\left\|\mathbf{W}_{r_{i}} \mathbf{h}_{b_{i}, r_{i}}\right\|^{2}\right.\right. & \left.\left.+\sigma^{2} \operatorname{tr}\left\{\mathbf{W}_{r_{i}}^{H} \mathbf{W}_{r_{i}}\right\}\right)\right) d \mathcal{E} \\
& -\oint_{\mathcal{R}} \sum_{i=1}^{N} \lambda_{i}(\mathcal{E})\left(\sum_{j=1}^{N}\left(\eta_{i, j}\left|\mathbf{h}_{r_{j}, s_{i}}^{H} \mathbf{W}_{r_{j}} \mathbf{h}_{b_{j}, r_{j}}\right|^{2}-\sigma^{2}\left\|\mathbf{h}_{r_{j}, s_{i}}^{H} \mathbf{W}_{r_{j}}\right\|^{2}\right)-\sigma^{2}\right) d \mathcal{E}
\end{aligned}
$$

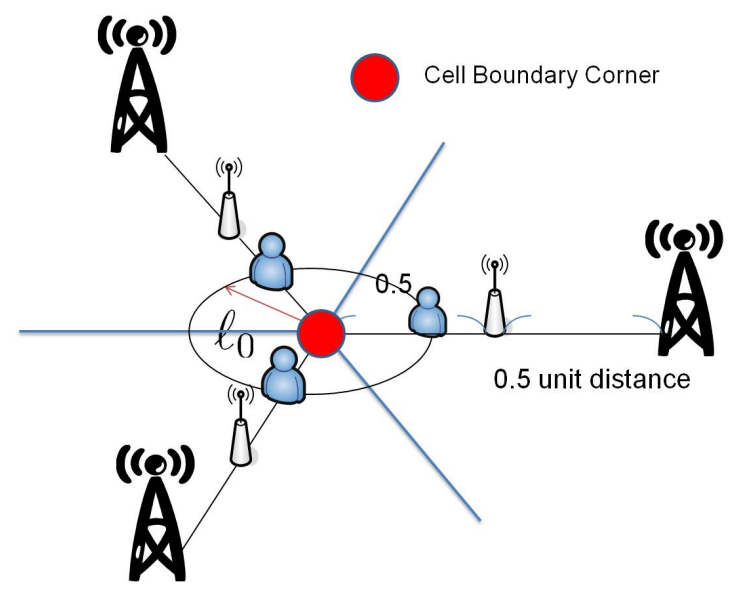

Fig. 3. An illustration of a three-cell cluster. Each cell in a cluster includes one RS and one SS.

\section{Simulation Studies}

We assess the performance of the proposed BFers in a physical-layer simulation model for CoMP AF relaying. In simulations, each data packet in CoMP transmissions consists of 100 QPSK-modulated symbols, and is declared erroneous if any of its demodulated symbols are incorrect. The throughput is evaluated as the number of correctly received bits divided by the total number of channel uses in symbol transmissions, i.e. in bits/(channel use). The maximum number of ARQ rounds is limited to one as the improvement over two is not significant enough. The proposed robust BFer designs are compared with the non-robust ones from the perspectives of power consumptions and feasibilities of BFer syntheses.

When comparing performance of different designs under a total transmit power of $P_{0}$, BFers are synthesized with their design criteria's dual problems in the form of

$$
\max _{\left\{\mathbf{W}_{r_{i}}\right\}_{i=1}^{N}} \min _{i \in\{1, \ldots, N\}} \operatorname{SINR}_{i} \text { s.t. } P_{r} \leq P_{0} .
$$

According to [28], (28) is, in fact, the dual problem of SCPM, e.g. the SCPM-R criterion of (5), and can be solved efficiently via its SCPM dual, using a one-dimensional bisection search method. On the other hand, when contrasting to ARQ that has no assistance of relays, BSs do downlink CoMP retransmissions by themselves. The designs of BF vectors for BSs in this case still follow the criteria of (5) and (8) as pointed out in Section II-B.

A system topology of a cluster of $N=3$ is illustrated in Fig. 3. To evaluate the pathloss effects in radiation power, the noise variance $\sigma^{2}$ is normalized to 1 , and the distance from the BSs to their joint cellular boundary corner is defined as a unit distance. Under this simulation setting, the distances from

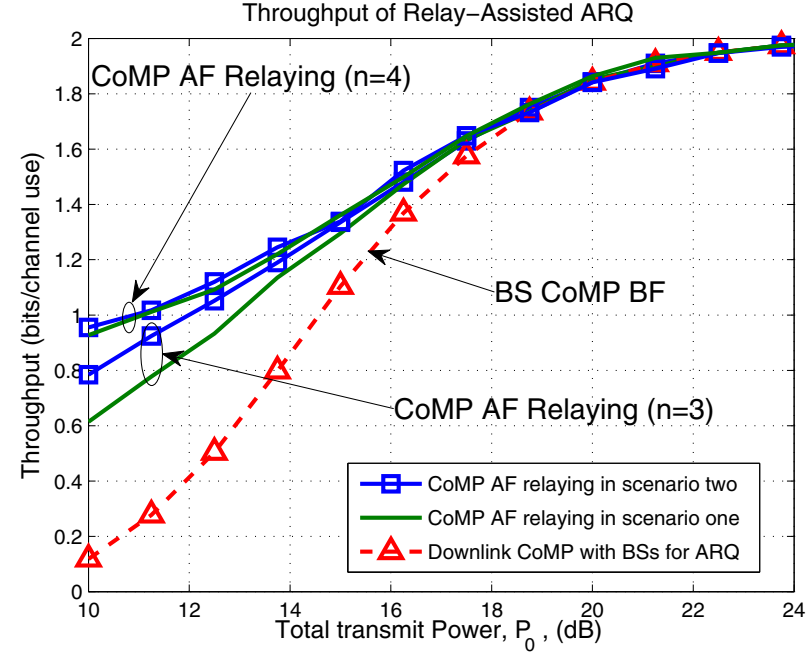

Fig. 4. Simulated throughput versus the total transmit power constraint, $P_{0}$, when $N=3$ and $\ell_{0}=0$.

the SSs to their joint boundaries are denoted by $\ell_{0}$ in the unit distance. The channel variance $\sigma_{i, j}^{2}$ between a transmit node $i$ and a receive node $j$ is defined as $\ell_{i, j}^{-n}$, where $n$ is the pathloss exponent and $\ell_{i, j}$ (in the unit distance) is the distance between nodes $i$ and $j$. Given a transmit power of $P_{i}$ for node $i$, the power received at node $j$ becomes $P_{i} \ell_{i, j}^{-n}$. For the conciseness of performance comparisons, all channels are considered to be block-faded. Namely, the channel coefficients remain unchanged within the duration of a transmission packet, and change randomly from packet to packet.

As described in the system model in Section II, the crosscell BS-to-RS MAI is ignored in BFer designs. To justify the rationality of this simplification, we first investigate a BFer's performance in two simulated scenarios. Scenario one models all MAIs, while scenario two ignores the cross-cell BS-to-RS MAI. The BFer design follows the criterion of (15) and its dual in the form of (28). The simulated throughput for the cases of $n=3$ and $n=4$ when $\ell_{0}=0$ are drawn w.r.t. $P_{0}$ in Fig. 4. In comparison, the throughput of only using BSs for ARQ is also shown in the figures. For brevity, this type of CoMP transmissions/retransmissions is referred to as the BS CoMP BF in the sequel. Apparently, the throughput gain with CoMP AF relaying is pronounced when $P_{0} \leq 15 \mathrm{~dB}$, which are typical values when all SSs are close to cellular boundaries. Furthermore, the throughput gains in scenario two are insignificant compared to that of scenario one.

The throughput of CoMP BF schemes versus $\ell_{0}$ when $P_{0}=$ $10 \mathrm{~dB}$ is presented in Fig. 5. The BFer designs still follow the criteria of (15) and (28). In simulations, SSs are set to move 


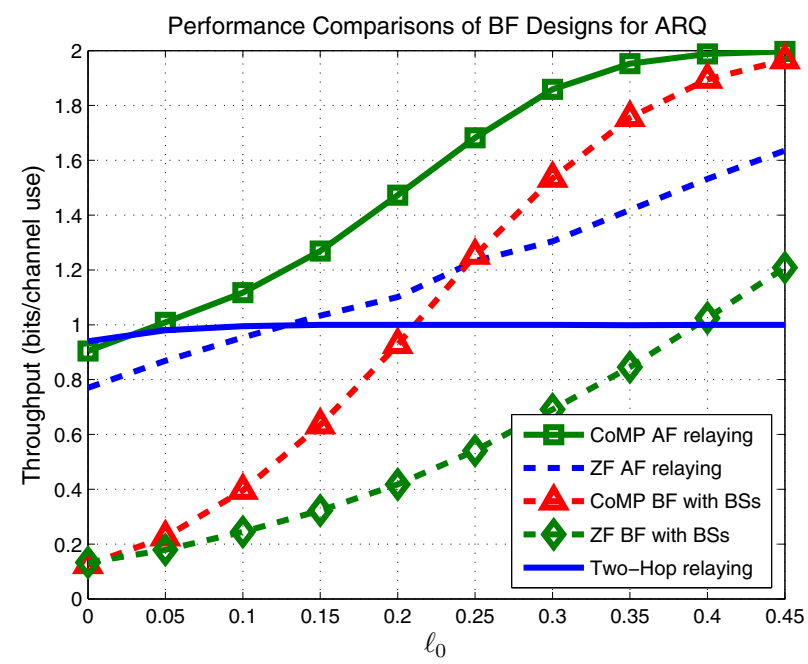

Fig. 5. Simulated throughput versus $\ell_{0}$ when $n=4, N=3$ and $P_{0}=$ $10 \mathrm{~dB}$.

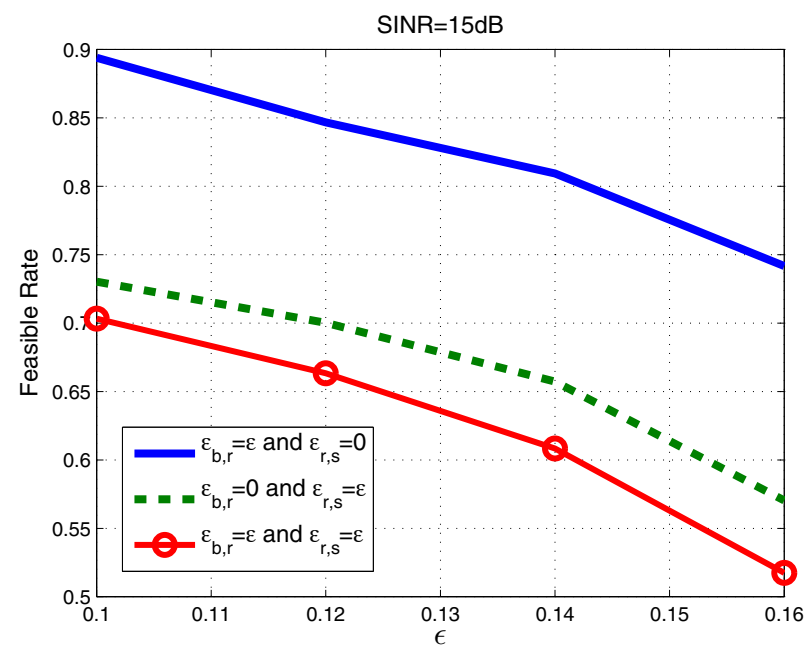

Fig. 6. The feasible rates of the non-robust BFer design to satisfy the SINR constraint of $\gamma_{0}=15 \mathrm{~dB}$ under different types of CSI uncertainties in CoMP AF relaying.

altogether from the joint boundary corner in Fig. 3 towards their BSs. Clearly, when $\ell_{0}$ are small, CoMP AF relaying has an obvious advantage over its counterpart of BS CoMP BF, and it also provides a gain larger than $0.2 \mathrm{bits} /($ channel use), compared to the zero-forcing ( $\mathrm{ZF}) \mathrm{AF}$ BF method that uses $\mathrm{ZF}$ BFers both at the BSs and the RSs. Additionally, throughput of using CoMP AF BF for two-hop relaying is also shown as a reference in the figure. A BS, in this case, first sends data towards its RS with a typical BF method, RSs of a cluster then relay the signals to SSs with the CoMP AF BF method.

We next verify the effectiveness of the proposed robust designs for CoMP AF relaying. All SSs are located at the joint cellular boundary, and the results are averaged over 1000 channel realizations. To assess the feasibility of a CoMP BFer design, the design is considered infeasible for a certain channel realization if it cannot satisfy all SSs' SINR requirements under a maximum power budget, $P_{\max }$, of $60 \mathrm{~dB}$. To contrast to the effectiveness of robust designs, performance of nonrobust designs is also evaluated in simulations.

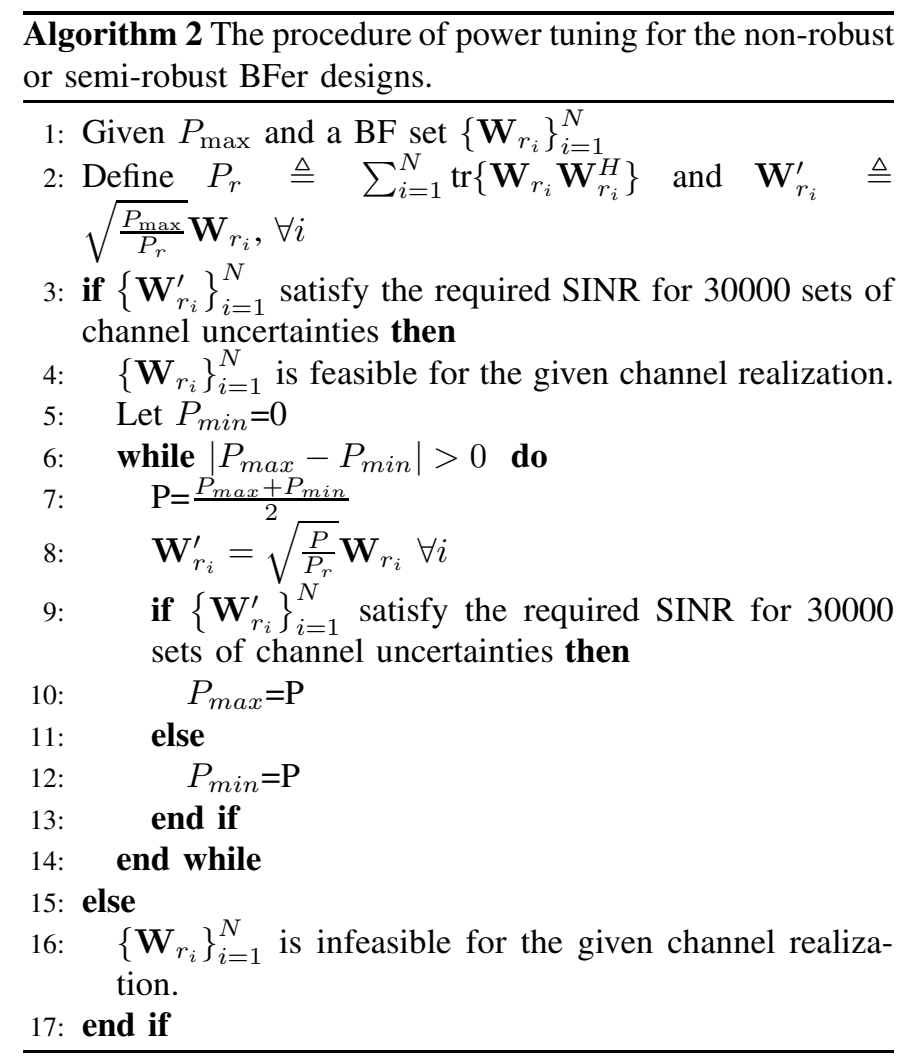

In non-robust designs, BFers are synthesized with (15) as if CSI feedbacks were perfect even if they are coupled with certain uncertainties. However, to ensure that the SINR constraint can be satisfied with the designs, the powers of the resultant BFers are scaled up to meet all SSs' SINR requirements in 30000 sets of CSI uncertainties whose $\left\|\mathbf{e}_{b_{i}, r_{i}}\right\| \leq \epsilon_{b, r}$ or $\left\|\mathbf{e}_{r_{j}, s_{i}}\right\| \leq \epsilon_{r, s}, \forall i, j$. To reduce the complexity in simulations, we consider in this case only a cluster of $N=2$ as shown in Fig. 2. A simple bisection method can be used to find the minimum sum power $P_{r}$ of the BFers. The algorithm is summarized in Algorithm 2.

This kind of non-robust design allows us to investigate the influences of $\mathbf{e}_{b_{i}, r_{i}}$ and $\mathbf{e}_{r_{j}, s_{i}}$, and the effects that might result from ignoring $\mathbf{e}_{b_{i}, r_{i}}$ in BFer designs. The feasible rates of this non-robust design under different types and degrees of uncertainties are presented in Fig. 6. The feasible rate is defined as the percentage of which a BFer design is feasible.

Clearly, the existence of $\mathbf{e}_{b_{i}, r_{i}}$ has limited impacts on the feasible rates, while $\mathbf{e}_{r_{j}, s_{i}}$ have devastating effects. This may be seen from the integral of $\oint \mathbf{h}_{b_{i}, r_{i}} \mathbf{h}_{b_{i}, r_{i}}^{H} d \mathbf{e}_{b_{i}, r_{i}}$ in (23) because $\oint \mathbf{h}_{b_{i}, r_{i}} \mathbf{h}_{b_{i}, r_{i}}^{H} d \mathbf{e}_{b_{i}, r_{i}}=\oint\left(\overline{\mathbf{h}}_{b_{i}, r_{i}}+\mathbf{e}_{b_{i}, r_{i}}\right)\left(\overline{\mathbf{h}}_{b_{i}, r_{i}}+\right.$ $\left.\mathbf{e}_{b_{i}, r_{i}}\right)^{H} d \mathbf{e}_{b_{i}, r_{i}} \approx \overline{\mathbf{h}}_{b_{i}, r_{i}} \overline{\mathbf{h}}_{b_{i}, r_{i}}^{H}+\oint \mathbf{e}_{b_{i}, r_{i}} \mathbf{e}_{b_{i}, r_{i}}^{H} d \mathbf{e}_{b_{i}, r_{i}} \lesssim$ $\overline{\mathbf{h}}_{b_{i}, r_{i}} \overline{\mathbf{h}}_{b_{i}, r_{i}}^{H}+\frac{1}{N} \epsilon_{b, r}^{2} \oint d \mathbf{e}_{b_{i}, r_{i}}$, supposed that the entries of $\mathbf{e}_{b_{i}, r_{i}}$ are statistically independent and have zero mean. This implies that the influence of $\mathbf{e}_{b_{i}, r_{i}}$ is small given that $\epsilon_{b, r}$ is small. It also in some sense validates the semi-robust criterion of (9) that ignores $\mathbf{e}_{b_{i}, r_{i}}$.

Performance of the SR-SCPM-R criterion of (9) is demonstrated in Fig. 7. Both solutions of the SDR method (17) and the QCQP-based scheme (21) are simulated for the case of $N=3, \epsilon_{b, r}=0$ and $\epsilon_{r, s}=0.1$. For comparison purposes, the results of the robust $\mathrm{BFer}$ design for $\mathrm{BS}$ CoMP $\mathrm{BF}$ are also 


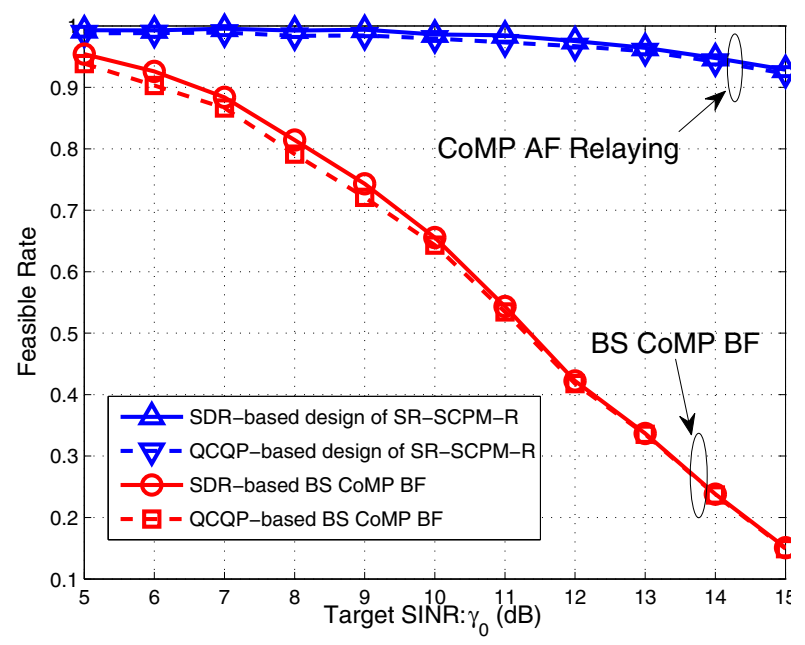

(a)

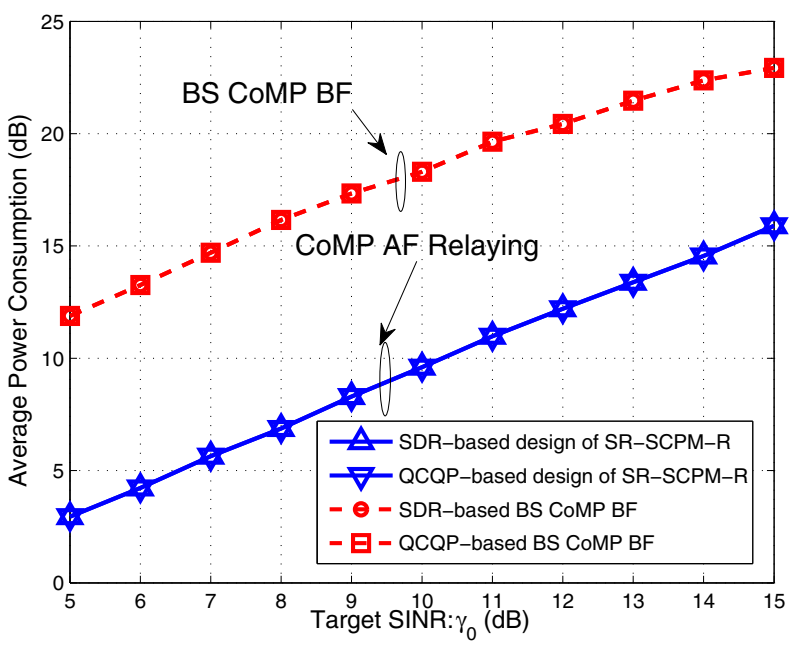

(b)

Fig. 7. Performance of two types of designs for the criterion of SR-SCPM-R, when $N=3$ and $\epsilon_{r, s}=0.1$.

shown in the figure. Clearly, the robust design for CoMP AF relaying, (since $\epsilon_{b, r}=0$ ), is much more power efficient and has a higher feasible rate to maintain the target SINR, $\gamma_{0}$, and, hence, QoS in ARQ. And the performance of the proposed QCQP scheme is very close to that of the SDR method. The results of the SDR method are obtained with the CVX solver [22]. As for the QCQP scheme, the design is considered infeasible once the number of iterations is greater than 100 . As can be seen from subplot (a), the feasible rates of the QCQP scheme are only slightly less than that of the SDR method. Results in subplot (b) also show that the power consumptions of these two schemes are indistinguishable once the QCQP scheme is feasible. In fact, the BF matrices obtained with the SDR method are optimal if they satisfy the rank-one condition. By inspection from simulations, the CVX solver seems always to yield the rank-one solutions. Similar observations have been reported in [26], [27] as well. This indicates that the proposed QCQP method and Algorithm 1 is an efficient solver.

The performance of the QCQP scheme for the R-SCPM-R criterion of (27) is presented in Fig. 8 when $\epsilon_{b, r}=\epsilon_{r, s}=0.2$. To verify the efficacy of the robust criterion of (27), results of the semi-robust criterion of (21) and the non-robust criterion of (15) are also shown in the figure. However, to ensure the robustness, the powers of these two designs are scaled up according to Algorithm 2. The feasible rates of the robust design (27) are higher than that of the design (21), and the power consumption of the design (27) is still slightly lower. Furthermore, the proposed robust/semi-robust BFer designs for CoMP AF relaying also provide significant gains either in feasible rates or on power consumptions when compared to the non-robust design of (15) or the robust design for BS CoMP BF.

\section{CONCLUSions}

Robust and semi-robust BFer designs were proposed for CoMP AF relaying to maintain the SINR in ARQ in downlink multi-cell networks. The proposed BF methods do not require realtime data exchanges among the different cells of a network, and are able to maintain the SINR under imperfect
CSI feedbacks. For the semi-robust criterion of SR-SCPM-R, the SDR method was shown by simulations to achieve a near optimal performance. And the QCQP method also presented a performance very close to the SDR one, and can handle the full robust criterion of R-SCPM-R. Although the optimal design for R-SCPM-R is still an open problem, the QCQP method presents to be an effective solution and is friendly for practical implementations. Simulations also showed that the downlink throughput close to the joint cellular boundaries of a multi-cell network can be effectively and power-efficiently improved with the proposed CoMP AF relaying method.

\section{ACKNOWLEDGEMENT}

The authors would like to thank Dr. Tsung-Hui Chang for his valuable suggestions on this work.

\section{APPENDIX}

Because the equalities $v_{i, i}=\frac{\epsilon_{r, s}}{\left\|h_{i, i}\right\|}$ yield trivial solutions, the Lagrangian for (21) follows

$$
\begin{aligned}
L= & \sum_{i=1}^{N} c_{i}\left\|\mathbf{w}_{r_{i}}\right\|^{2}-\sum_{i=1}^{N} \sum_{j=1}^{N} \tau_{j, i} \mathbf{w}_{r_{j}}^{H} \mathbf{G}_{j, i} \mathbf{w}_{r_{j}} \\
& -\sum_{i=1}^{N} \lambda_{i}\left(\frac{1}{\gamma_{0}} \mathbf{w}_{r_{i}}^{H} \mathbf{A}_{i} \mathbf{w}_{r_{i}}-\sum_{j=1, j \neq i}^{N} \mathbf{w}_{r_{j}}^{H} \mathbf{B}_{j, i} \mathbf{w}_{r_{j}}-\sigma^{2}\right)
\end{aligned}
$$

where $\mathbf{G}_{j, i} \triangleq v_{j, i}^{2}\left\|\overline{\mathbf{h}}_{r_{j}, s_{i}}\right\|^{2} \mathbf{I}_{N}-\overline{\mathbf{h}}_{r_{j}, s_{i}} \overline{\mathbf{h}}_{r_{j}, s_{i}}^{H}, \forall i, j$. The KKT conditions for (21) is given by

1) Feasibility: This includes $\lambda_{i}, \tau_{i, j} \geq 0, v_{i, i} \geq \frac{\epsilon_{r, s}}{\left\|\mathbf{h}_{r_{i}, s_{i}}\right\|}, v_{j, i} \geq$ $0, \mathbf{w}_{r_{j}}^{H} \mathbf{G}_{j, i} \mathbf{w}_{r_{j}} \geq 0, \forall i, j$, and

$$
\frac{1}{\gamma_{0}} \mathbf{w}_{r_{i}}^{H} \mathbf{A}_{i} \mathbf{w}_{r_{i}} \geq \sum_{j=1, j \neq i}^{N} \mathbf{w}_{r_{j}}^{H} \mathbf{B}_{j, i} \mathbf{w}_{r_{j}}+\sigma^{2}, \forall i .
$$

2) Complementary Slackness: This includes $\left|\overline{\mathbf{h}}_{r_{j}, s_{i}}^{H} \mathbf{w}_{r_{j}}\right|^{2}=$ $v_{j, i}^{2}\left\|\overline{\mathbf{h}}_{r_{j}, s_{i}}\right\|^{2}\left\|\mathbf{w}_{r_{j}}\right\|^{2}, \forall i, j$, and

$$
\frac{1}{\gamma_{0}} \mathbf{w}_{r_{i}}^{H} \mathbf{A}_{i} \mathbf{w}_{r_{i}}=\sum_{j=1, j \neq i}^{N} \mathbf{w}_{r_{j}}^{H} \mathbf{B}_{j, i} \mathbf{w}_{r_{j}}+\sigma^{2}, \forall i .
$$




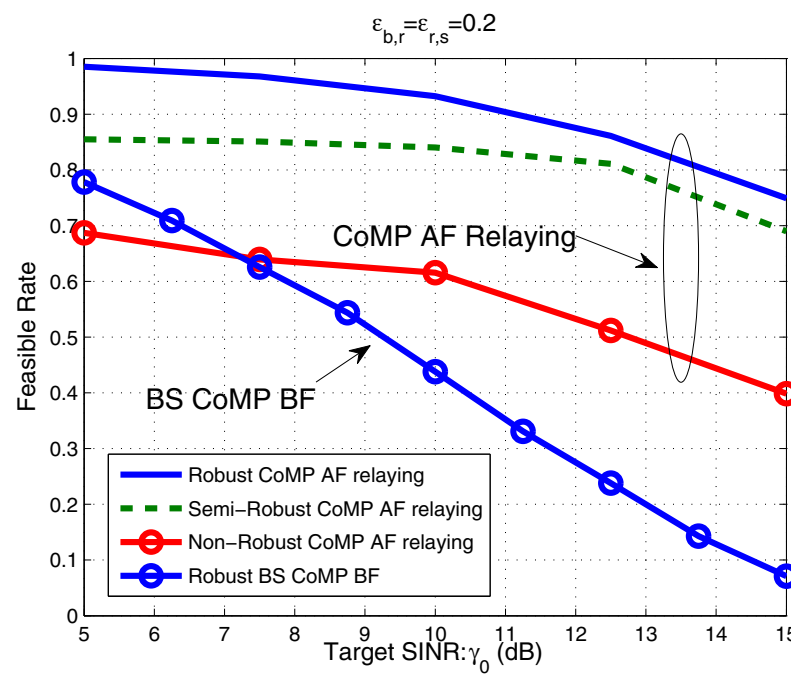

(a)

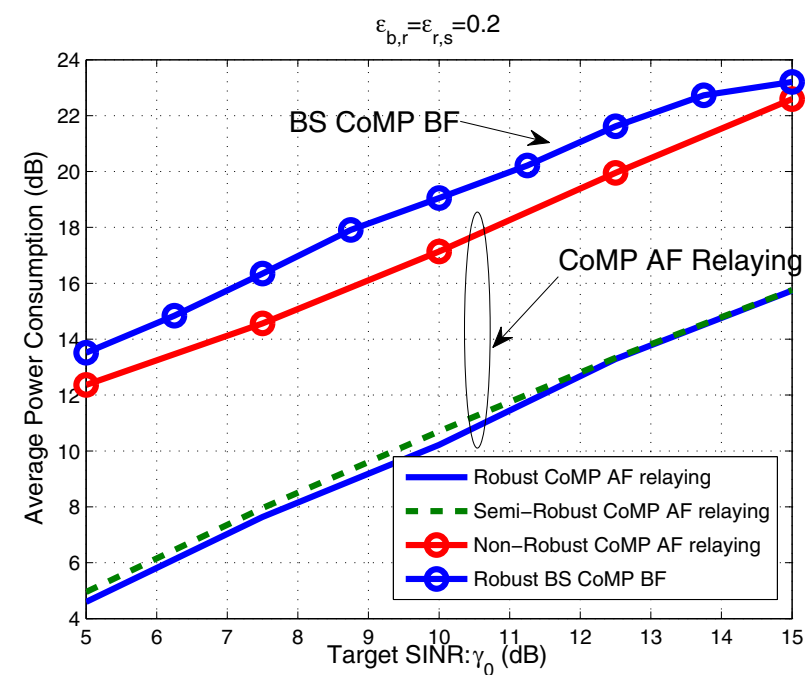

(b)

Fig. 8. Performance of various types of designs versus $\gamma_{0}$ when $N=2$ and $\epsilon_{b, r}=\epsilon_{r, s}=0.2$.

3) Zero derivative: The zero derivatives of (29) w.r.t. $v_{i, j}$ yield $\tau_{i, i}=\frac{\epsilon_{r, s} d_{i} \lambda_{i}}{\gamma_{0} v_{i, i}\left\|\mathbf{h}_{r_{i}, s_{i}}\right\|}, \forall i$, and $\tau_{j, i}=\frac{\epsilon_{r, s} c_{j} \lambda_{i}}{v_{j, i}\left\|\mathbf{h}_{r_{j}, s_{i}}\right\|}, \forall j \neq i$. Besides, the zero derivatives of (29) w.r.t. $\mathbf{w}_{r_{i}}$ return $\Psi_{i} \mathbf{w}_{r_{i}}=$ $\mathbf{0}$ where $\boldsymbol{\Psi}_{i} \triangleq c_{i} \mathbf{I}_{N}-\frac{\lambda_{i}}{\gamma_{0}}\left(\mathbf{A}_{i}\right)+\sum_{m=1, m \neq i}^{N} \lambda_{m} \mathbf{B}_{i, m}-$ $\sum_{m=1}^{N} \tau_{i, m} \mathbf{G}_{i, m}$. This implies $\left(\boldsymbol{\Psi}_{i}+\frac{\lambda_{i} d_{i}}{\gamma_{0}} \overline{\mathbf{h}}_{r_{i}, s_{i}} \overline{\mathbf{h}}_{r_{i}, s_{i}}^{H}\right) \mathbf{w}_{r_{i}}=$ $\frac{\lambda_{i} d_{i}}{\gamma_{0}} \overline{\mathbf{h}}_{r_{i}, s_{i}} \overline{\mathbf{h}}_{r_{i}, s_{i}}^{H} \mathbf{w}_{r_{i}}$. Define $\boldsymbol{\Phi}_{i, i} \triangleq \boldsymbol{\Psi}_{i}+\frac{\lambda_{i} d_{i}}{\gamma_{0}}\left(\overline{\mathbf{h}}_{r_{i}, s_{i}} \overline{\mathbf{h}}_{r_{i}, s_{i}}^{H}\right)$, we have

$$
\mathbf{w}_{r_{i}}=\frac{\lambda_{i} d_{i}}{\gamma_{0}}\left(\overline{\mathbf{h}}_{r_{i}, s_{i}}^{H} \mathbf{w}_{r_{i}}\right) \boldsymbol{\Phi}_{i, i}^{-1} \overline{\mathbf{h}}_{r_{i}, s_{i}}, \forall i .
$$

Replacing $\mathbf{w}_{r_{i}}$ in (31) with (32) results in

$$
\boldsymbol{\Gamma}=\sigma^{2} \mathbf{F}^{-1} \mathbf{1}_{N}
$$

where $\boldsymbol{\Gamma} \triangleq\left[\left|\delta_{1}\right|^{2}, \ldots,\left|\delta_{N}\right|^{2}\right]^{T}$ with $\delta_{i} \triangleq \overline{\mathbf{h}}_{r_{i}, s_{i}}^{H} \mathbf{w}_{r_{i}}$ and $\mathbf{1}_{N}$ is a $N \times 1$ vector whose entries all equal to one. The diagonal entries of $\mathbf{F}$ are defined as $[\mathbf{F}]_{i, i} \triangleq\left(\frac{\lambda_{i} d_{i}}{r_{0}}\right)^{2} \overline{\mathbf{h}}_{r_{i}, s_{i}}^{H} \boldsymbol{\Phi}_{i, i}^{-1}\left(\frac{\mathbf{A}_{i}}{r_{0}}\right) \boldsymbol{\Phi}_{i, i}^{-1} \overline{\mathbf{h}}_{r_{i}, s_{i}}, \forall i$. And $[\mathbf{F}]_{i, j} \triangleq$ $-\left(\frac{\lambda_{j} d_{j}}{r_{0}}\right)^{2} \overline{\mathbf{h}}_{r_{j}, s_{j}}^{H} \boldsymbol{\Phi}_{j, j}^{-1} \mathbf{B}_{j, i} \boldsymbol{\Phi}_{j, j}^{-1} \overline{\mathbf{h}}_{r_{j}, s_{j}}$ are its off diagonal entries, $\forall j \neq i$.

Observe from (21) that if $\hat{\mathbf{w}}_{r_{i}}$ are optimal, then $e^{j \theta_{i}} \hat{\mathbf{w}}_{r_{i}}$ are optimal as well, where $\theta_{i}$ are arbitrary phases. Therefore, WLOG, we can have $\delta_{i}=\overline{\mathbf{h}}_{r_{i}, s_{i}}^{H} \mathbf{w}_{r_{i}} \geq 0$. Consequently, $\delta_{i}$ can be solved immediately from (33). The $\mathrm{BF}$ vectors, $\mathbf{w}_{r_{i}}$, can then be obtained as long as $\lambda_{i}$, $v_{j, i}$, and, hence, $\tau_{j, i}$, are known. To this end, we first multiply the both sides of (32) by $\overline{\mathbf{h}}_{r_{i}, s_{i}}^{H}$ to yield $\lambda_{i}=$ $\frac{\gamma_{0}}{d_{i} \overline{\mathbf{h}}_{r_{i}, s_{i}}^{H} \mathbf{\Phi}_{i, i}^{-1} \overline{\mathbf{h}}_{r_{i}, s_{i}}}$ if $\overline{\mathbf{h}}_{r_{i}, s_{i}}^{H} \mathbf{w}_{r_{i}} \neq 0, \forall i$. Besides, (32) itself also returns $\left\|\mathbf{w}_{r_{i}}\right\|^{2}=\left(\frac{\lambda_{i} d_{i}}{\gamma_{0}}\right)^{2} \overline{\mathbf{h}}_{r_{i}, s_{i}}^{H} \boldsymbol{\Phi}_{i, i}^{-2} \overline{\mathbf{h}}_{r_{i}, s_{i}}\left|\overline{\mathbf{h}}_{r_{i}, s_{i}}^{H} \mathbf{w}_{r_{i}}\right|^{2}$. Together with the complementary slackness condition of $\left|\overline{\mathbf{h}}_{r_{i}, s_{i}}^{H} \mathbf{w}_{r_{i}}\right|^{2}=v_{i, i}^{2}\left\|\overline{\mathbf{h}}_{r_{i}, s_{i}}\right\|^{2}\left\|\mathbf{w}_{r_{i}}\right\|^{2}$, we then obtain $v_{i, i}=$ $\frac{\gamma_{0}}{\lambda_{i} d_{i}\left\|\overline{\mathbf{h}}_{r_{i}, s_{i}}\right\|\left\|\mathbf{\Phi}_{i, i}^{-1} \overline{\mathbf{h}}_{r_{i}, s_{i}}\right\|}, \forall i$.

On the other hand, by subtracting $\lambda_{j} c_{i} \overline{\mathbf{h}}_{r_{i}, s_{j}} \overline{\mathbf{h}}_{r_{i}, s_{j}}^{H} \mathbf{w}_{r_{i}}$ from the both sides of $\boldsymbol{\Psi}_{i} \mathbf{w}_{r_{i}}=\mathbf{0}$, we obtain another equation $\left(\boldsymbol{\Psi}_{i}-\lambda_{j} c_{i} \overline{\mathbf{h}}_{r_{i}, s_{j}} \overline{\mathbf{h}}_{r_{i}, s_{j}}^{H}\right) \mathbf{w}_{r_{i}}=-\lambda_{j} c_{i} \overline{\mathbf{h}}_{r_{i}, s_{j}} \overline{\mathbf{h}}_{r_{i}, s_{j}}^{H} \mathbf{w}_{r_{i}}$, which results in $\mathbf{w}_{r_{i}}=-\left(\lambda_{j} c_{i}\right) \boldsymbol{\Phi}_{i, j}^{-1} \overline{\mathbf{h}}_{r_{i}, s_{j}} \overline{\mathbf{h}}_{r_{i}, s_{j}}^{H} \mathbf{w}_{r_{i}}$ and, hence, $\left\|\mathbf{w}_{r_{i}}\right\|^{2}=\left(\lambda_{j} c_{i}\right)^{2} \overline{\mathbf{h}}_{r_{i}, s_{j}}^{H} \boldsymbol{\Phi}_{i, j}^{-2} \overline{\mathbf{h}}_{r_{i}, s_{j}}\left|\overline{\mathbf{h}}_{r_{i}, s_{j}}^{H} \mathbf{w}_{r_{i}}\right|^{2}$ with $\boldsymbol{\Phi}_{i, j} \triangleq$
$\boldsymbol{\Psi}_{i}-\lambda_{j} c_{i} \overline{\mathbf{h}}_{r_{i}, s_{j}} \overline{\mathbf{h}}_{r_{i}, s_{j}}^{H}, \forall j \neq i$. By the complementary slackness condition of $\left|\overline{\mathbf{h}}_{r_{i}, s_{j}}^{H} \mathbf{w}_{r_{i}}\right|^{2}=v_{i, j}^{2}\left\|\overline{\mathbf{h}}_{r_{i}, s_{j}}\right\|^{2}\left\|\mathbf{w}_{r_{i}}\right\|^{2}$, we then obtain $v_{i, j}=\frac{1}{\lambda_{j} c_{i}\left\|\overline{\mathbf{h}}_{r_{i}, s_{j}}\right\|\left\|\boldsymbol{\Phi}_{i, j}^{-1} \overline{\mathbf{h}}_{r_{i}, s_{j}}\right\|}, \forall j \neq i$.

Finally, the condition of $\boldsymbol{\Psi}_{i} \mathbf{w}_{r_{i}}=\mathbf{0}$ directly yields the optimal objective $P_{r}=\sum_{i=1}^{N} \sigma^{2} \lambda_{i}$.

\section{REFERENCES}

[1] R. Irmer, H. Droste, P. Marsch, M. Grieger, G. Fettweis, S. Brueck, H.-P. Mayer, L. Thiele, and V. Jungnickel, "Coordinated multipoint: concepts, performance, and field trial results," IEEE Commun. Mag., vol. 49, no. 2, pp. 102-111, Feb. 2011.

[2] R. A. DiFazio and P. J. Pietraski, "The bandwidth crunch: can wireless technology meet the skyrocketing demand for mobile data?" in 2011 IEEE Syst., Appl. Technol. Conf.

[3] K. Zheng, B. Fan, Z. Ma, G. Liu, X. Shen, and W. Wang, "Multihop cellular networks toward LTE-advanced," IEEE Veh. Technol. Mag., vol. 4, no. 3, pp. 40-47, Sep. 2009.

[4] Y. Yang, H. Hu, J. Xu, and G. Mao, "Relay technologies for WiMAX and LTE-Advanced mobile systems," IEEE Commun. Mag., vol. 47, no. 10, pp. 100-105, Oct. 2009.

[5] H. Dahrouj and W. Yu, "Coordinated beamforming for the multicell multi-antenna wireless system," IEEE Trans. Wireless Commun., vol. 9 , no. 5, pp. 1748-1759, May 2010.

[6] G. Caire, S. A. Ramprashad, and H. C. Papadopoulos, "Rethinking network MIMO: cost of CSIT, performance analysis, and architecture comparisons," in 2010 Inf. Theory Appl. Workshop.

[7] H. Huang, M. Trivellato, A. Hottinen, M. Shafi, P. Smith, and R. Valenzuela, "Increasing downlink cellular throughput with limited network MIMO coordination," IEEE Trans. Wireless Commun., vol. 8, no. 6, pp. 2983-2989, June 2009.

[8] P. Marsch and G. Fettweis, "Static clustering for cooperative multi-point (CoMP) in mobile communications," in 2011 IEEE International Conf. Commun.

[9] V. Kotzsch and G. Fettweis, "On synchronization requirements and performance limitations for CoMP systems in large cells," in 2011 International Workshop Multi-Carrier Syst. Solutions.

[10] J. Zhang, G. Liu, F. Zhang, L. Tian, N. Sheng, and P. Zhang, "Advanced international communications," IEEE Veh. Technol. Mag., vol. 6, no. 2, pp. 92-100, June 2011.

[11] V. M. Stencel and P. A. Frank, "LTE Advanced-A further evolutionary step for next generation mobile networks," in 2010 International Conf. Radioelektronika.

[12] A. Tajer, N. Prasad, and X. Wang, "Robust linear precoder design for multi-cell downlink transmission," IEEE Trans. Signal Process., vol. 59, no. 1, pp. 235-251, Jan. 2011.

[13] N. Jindal, "MIMO broadcast channels with finite-rate feedback," IEEE Trans. Inf. Theory, vol. 52, no. 11, pp. 5045-5060, Nov. 2006. 
[14] M. Botros and T. N. Davidson, "Convex conic formulations of robust downlink precoder designs with quality of service constraints," IEEE J. Sel. Topics Signal Process., vol. 1, no. 4, pp. 714-724, Dec. 2007.

[15] N. Vucic and H. Boche, "Robust QoS-constrained optimization of downlink multiuser MISO systems," IEEE Trans. Signal Process., vol. 57, no. 2, pp. 714-725, Feb. 2009.

[16] D. Ponukumati, F. Gao, and L. Fan, "Robust general rank precoding design for amplify-and-forward relay network," in Proc. 2010 IEEE Global Telecommun. Conf., pp. 1-5.

[17] B. K. Chalise and L. Vandendorpe, "Optimization of MIMO relays for multipoint-to-multipoint communications: nonrobust and robust designs," IEEE Trans. Signal Process., vol. 58, no. 12, pp. 6355-6368, Dec. 2010.

[18] P. Rost, G. Fettweis, and J. Laneman, "Opportunities, constraints, and benefits of relaying in the presence of interference," in Proc. 2009 IEEE International Conf. Commun., pp. 1-5.

[19] A. Papadogiannis, A. G. Burr, and M. Tao, "On the maximum achievable sum-rate of interfering two-way relay channels," IEEE Commun. Lett., vol. 16, no. 1, pp. 72-75, Jan. 2012.

[20] S. Boyd and L. Vandenberghe, Convex Optimization. Cambridge University Press, 2004.

[21] Z.-Q. Luo, W.-K. Ma, A. M.-C. So, Y. Ye, and S. Zhang, "Semidefinite relaxation of quadratic optimization problems," IEEE Signal Process. Mag., vol. 27, no. 3, pp. 20-34, May 2010.

[22] M. Grant and S. Boyd, "CVX: Matlab software for disciplined convex programming, version 1.21." Available: http://cvxr.com/cvx, Feb. 2011.

[23] J. F. Sturm, "Using SeDuMi 1.02, a MATLAB toolbox for optimization over symmetric cones,", Optimization Methods Software, vol. 11-12, pp. 625-653, 1999.

[24] H. Ochiai, P. Mitran, and V. Tarokh, "Variable rate two phase collaborative communication protocols for wireless networks," IEEE Trans. Inf. Theory, vol. 52, no. 9, pp. 4299-4313, Sep. 2006.

[25] A. Papadogiannis, H. J. Bang, D. Gesbert, and E. Hardouin, "Efficient selective feedback design for multicell cooperative networks," IEEE Trans. Veh. Technol., vol. 60, no. 1, pp. 196-205, Jan. 2011.

[26] G. Zheng, K.-K. Wong, and T.-S. Ng, "Robust linear MIMO in the downlink: a worst-case optimization with ellipsoidal uncertainty regions," EURASIP J. Appl. Signal Process., vol. 2008, pp. 1-15, June 2008.

[27] K.-Y. Wang, T.-H. Chang, W.-K. Ma, and C.-Y. Chi, "A semidefinite relaxation based conservative approach to robust transmit beamforming with probabilistic SINR constraints," in Proc. 2010 EUSIPCO.

[28] A. Wiesel, Y. C. Eldar, and S. Shamai, "Linear precoding via conic optimization for fixed MIMO receivers," IEEE Trans. Signal Process., vol. 54, no. 1, pp. 161-176, Jan. 2006

[29] E. de Klerk, Aspects of Semidefinite Programming: Interior Point Algorithms and Selected Applications. Kluwer Academic Publishers, 2002.

[30] S.-J. Kim, A. Magnani, A. Mutapcic, S. P. Boyd, and Z.-Q. Luo, "Robust beamforming via worst-case SINR maximization," IEEE Trans. Signal Process., vol. 56, no. 4, pp. 1539-1547, Apr. 2008.

[31] V. Havary-Nassab, S. Shahbazpanahi, A. Grami, and Z.-Q. Luo, "Distributed beamforming for relay networks based on second-order statistics of the channel state information," IEEE Trans. Signal Process., vol. 56, no. 9, pp. 4306-4316, Sep. 2008.

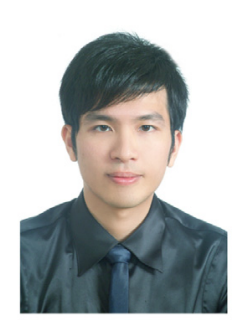

Chun-I Kuo was born in Changhua, Taiwan. He received the B.S. and M.S. degrees in communication engineering from National Chiao Tung University (NCTU), Hsinchu, Taiwan, in 2009 and 2011, respectively. During his graduate studies from 2009 to 2011, he was a member of the Mobile Broadband Wireless Communication Laboratory, NCTU. He is currently with MStar Semiconductor, Inc., Taiwan, where he is a Senior R\&D Engineer working on algorithms design for mobile receivers. His research interest is in the application of signal processing to

inner receiver design.

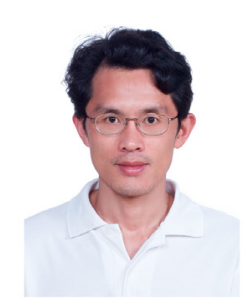

Sau-Hsuan Wu received the B.S. and the M.S. degrees from National Cheng Kung University, Taiwan in 1990 and 1993, respectively, both in Engineering Science. From 1993 to 1995, he served in the Army of Taiwan, and from 1995 to 1999 , he worked as a circuit and system design engineer in Taiwan. In 2003, he received the Ph.D. degree in Electrical Engineering from the University of Southern California, Los Angeles. From 2003 to 2004, he was a postdoctoral research fellow at the Department of Electrical Engineering, University of Southern California, Los Angeles. From 2004 to 2005, he served as a technical consultant for Winbond Electronics Corporation America, developing wireless MIMO-OFDM products. Since 2005, he has been an Assistant Professor at the Department of Electrical and Computer Engineering, National Chiao Tung University, Taiwan. His research interest lies in the application of signal processing theory to wireless communication systems.

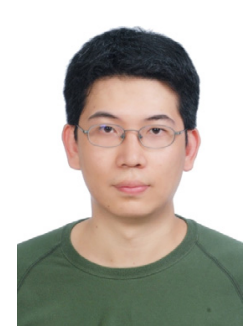

Chun-Kai Tseng received the B.S. degree in Electrical Engineering from National Central University, Taiwan, in 2003 and the M.S. degree in Communications Engineering from National Chung Cheng University, in 2008. He currently is a Ph.D. student at the Institute of Communications Engineering, National Chiao Tung University (NCTU), Taiwan, and is a member of the Mobile Broadband Wireless Communication Laboratory, NCTU. His research interests are in the theoretical analysis on cooperative communications, including relaying techniques and 